\title{
A Cost and Passenger Responsible Optimization Method for the Operation Plan of Additional High-Speed Trains in a Peak Period
}

\author{
Yutong Liu, Chengxuan Cao $(\mathbb{D}$, Ziyan Feng $(\mathbb{D}$, and Yaling Zhou \\ State Key Laboratory of Rail Traffic Control and Safety, Beijing Jiaotong University, Beijing 100044, China \\ Correspondence should be addressed to Chengxuan Cao; cxcao@bjtu.edu.cn
}

Received 4 July 2019; Revised 7 January 2020; Accepted 8 February 2020; Published 10 March 2020

Academic Editor: Paola Pellegrini

Copyright ( 2020 Yutong Liu et al. This is an open access article distributed under the Creative Commons Attribution License, which permits unrestricted use, distribution, and reproduction in any medium, provided the original work is properly cited.

\begin{abstract}
In the peak period of a railway system, operators typically add additional trains to provide increased capacity to satisfy the increasing passenger demand. The paper proposes a new optimization framework for designing the operation plan, which includes the number of additional trains, train type, stop plan, and timetable, for additional trains in a peak period. A space-time network representation is used to obtain a feasible primary operation plan by finding a set of feasible space-time paths in the spacetime network. Considering simultaneously the passenger demand and the trains' total travel times, we formulate a biobjective integer programming model for generating a cost and passenger responsible primary operation plan. A set of loading capacity constraints are formulated in the model to guarantee a suitable loading capacity for each station's passenger demand and better service for passengers. The CPLEX solver is used to solve the proposed model and to generate the optimal operation plan. Two sets of numerical experiments are conducted on a small-scale rail corridor and on the Wuhan-Guangzhou rail corridor to evaluate the performance of the proposed method. The results of the experiments show that the primary operation plan can be obtained within an acceptable computation time.
\end{abstract}

\section{Introduction}

With the development of science, technology, and economics, numerous kilometers of high-speed railway have been constructed in some countries to meet increasing passenger demands. The efficient operation of high-speed trains has become an attractive issue in recent years. A plan that is related to the operation of high-speed trains is called operation plan, for example, a plan for the number of trains, a timetable, a stopping plan, a plan for rolling stock, or a crew plan. A well-designed operation plan can reduce traveling times and provide better service for passengers. From the perspective of the railway company, a satisfactory operation plan results in lower operational cost and lower energy consumption. This paper aims to solve the problem of the operation plan for additional high-speed trains in peak periods.

1.1. Motivation. The primary motivation of this study is the insufficient railway transport capacity during peak periods.
Passenger demand for rail transport fluctuates. The demand remains at a high level in some periods, which are called peak periods, while the demand is at a normal level in other periods, which are called off-peak periods. Consider rail transport in China as an example: The passenger demand surge is very strong in the period of the Chinese Spring Festival. The number of passengers in the month of the Chinese Spring Festival was 413 million in 2019. Although the passenger demand fluctuation was considered in the initial stage of the operation plan design, there were still passengers who could not buy tickets to take a high-speed train during the peak period due to the increased passenger demand. In 2019, the number of passengers in the period of the Chinese Spring Festival increased by $8.3 \%$ over the previous year. The addition of trains has become a necessary strategy of railway companies for increasing the transport capacity in peak periods. A significant issue that is faced by railway operators in practical operations is the design of a satisfactory operation plan for additional trains in peak periods in a short time horizon that can satisfy large 
passenger demand and enhance the utilization efficiency of the transport resource.

The railway organizational structure differs among countries. In this paper, the rail undertaking (which operates the trains) and the infrastructure manager (which constructs the timetable) are the same organization. The organization is called the railway company in this paper. The railway company formulates a long-term operation plan via a complex planning process at the beginning of the construction of a railway corridor. Once the operation plan is established, the railway company intends to use the operation plan for a long time with little modification. Robenek et al. [1] developed a flow chart, which is presented as Figure 1, that illustrates the railway company's process of designing a complete operation plan according to the description in Caprara et al. [2].

The railway organizational structure differs among countries. In this paper, the rail undertaking (which operates the trains) and the infrastructure manager (which constructs the timetable) are the same organization. The organization is called the railway company in this paper. The railway company formulates a long-term operation plan via a complex planning process at the beginning of the construction of a railway corridor. Once the operation plan is established, the railway company intends to use the operation plan for a long time with little modification. Lusby et al. [3] developed a flow chart, which is presented as Figure 1, that illustrates the railway company's process of designing a complete operation plan. However, operators typically adopt the strategy of adding trains several weeks in advance. Designing the operation plan for additional trains step by step as Figure 1 will take a long time, especially the strategic and tactic stages. Aiming to produce an operation plan for additional trains in short-term horizon, we are interested in how to design a primary operation plan including the number of additional trains, train type, stop plan, and timetable. In the process of Figure 1, the decisions of the number of trains, train type, and stop plan are in the line planning, while timetable generation is in the tactical level of decision-making, which is restricted by the results of line planning. Generating a comprehensive operation plan of them on the tactic level can raise efficiency. The content of the primary plan in our study is highlighted in red in Figure 1. Generating the primary operation plan on the tactic level can handle travel rush in the short-term and assist the existing operation plan. Designing a primary operation plan, which includes the number of trains, train type, stop plan, and timetable, for additional trains, has not attracted sufficient attention, and we hereinafter shall address this issue formally.

1.2. Literature Review. The problem of this paper is the problem of scheduling additional trains. In this literature review, we first overview the literatures on the problem of scheduling additional trains. The literatures on the problem of scheduling additional trains directly are rare because the problem of scheduling additional trains has not been concerned widely. In addition, the purpose of our study is to obtain a comprehensive operation plan for additional trains. The decision variables in our problem are the variables of the number of additional trains, train type, stop plan, and timetable. These variables also are studied in the Line planning problem (LPP) and Train timetable problem (TTP). Thus, our problem falls in the broad category of LPP and TTP. The related literatures on LPP and TTP are also overviewed in this section.

The problem of adding additional trains has not attracted much attention. Only a few studies [4-6] focused directly on the problem of designing an operation plan or timetable for additional trains. Burdett and Kozan [4] considered the problem of scheduling additional trains as a hybrid job shop scheduling problem with time window constraints. The original timetable was fine-tuned according to the operations and the demand of various customers or operators. A constructive algorithm and a simulated annealing approach were used to solve this problem. Cacchiani et al. [5] studied the problem of scheduling additional freight trains in a timetable of existing passenger trains under the constraint that the timetable of passenger trains cannot be changed. An integer programming model was established, in which an ideal timetable of additional freight trains was specified and adjusted according to constraints that ensure safe operation. The objective was to add as many trains as possible and to minimize the difference between the actual timetable of additional freight trains and the ideal timetable. Gao et al. [6] considered the problem of scheduling additional trains on a high-speed rail corridor where only passenger trains were run. To add more trains, the timetable of the original trains may need to be modified in that study. A biobjective mixedinteger linear programming model was formulated, of which the objectives were to minimize the total travel times of the additional trains and to adjust the timetable of the original trains. Although all three of these studies considered the problem of adding additional trains, they did not consider the passenger demand. In addition, these studies focused on the timetable of additional trains without considering other operation plans; for example, the number of trains, stop plan, has not been studied. Especially, the suitable number of additional trains according to passenger demand is an important issue, which is not attractive widely. This gap is addressed herein.

Generally, the line planning and timetable generation are designed separately. The line planning operation plan is generated first; the timetable is specified according to it. Pouryousef and Lautala [7] presented the hybrid simulation framework to improve the capacity utilization of the railway and timetable. The hybrid simulation experiments in this paper were implemented based on two types of developed simulation software, that is, timetable simulation system and nontimetable simulation. The hybrid simulation approach is to make use of the complementary features of nontimetable and timetable, and use the output from a simulation system as input for the other simulation system. Although the hybrid simulation framework can obtain an operation plan of timetable and nontimetable simultaneously, the approach needs long running times and a computer with very high performance. The joint modeling method is used in this 


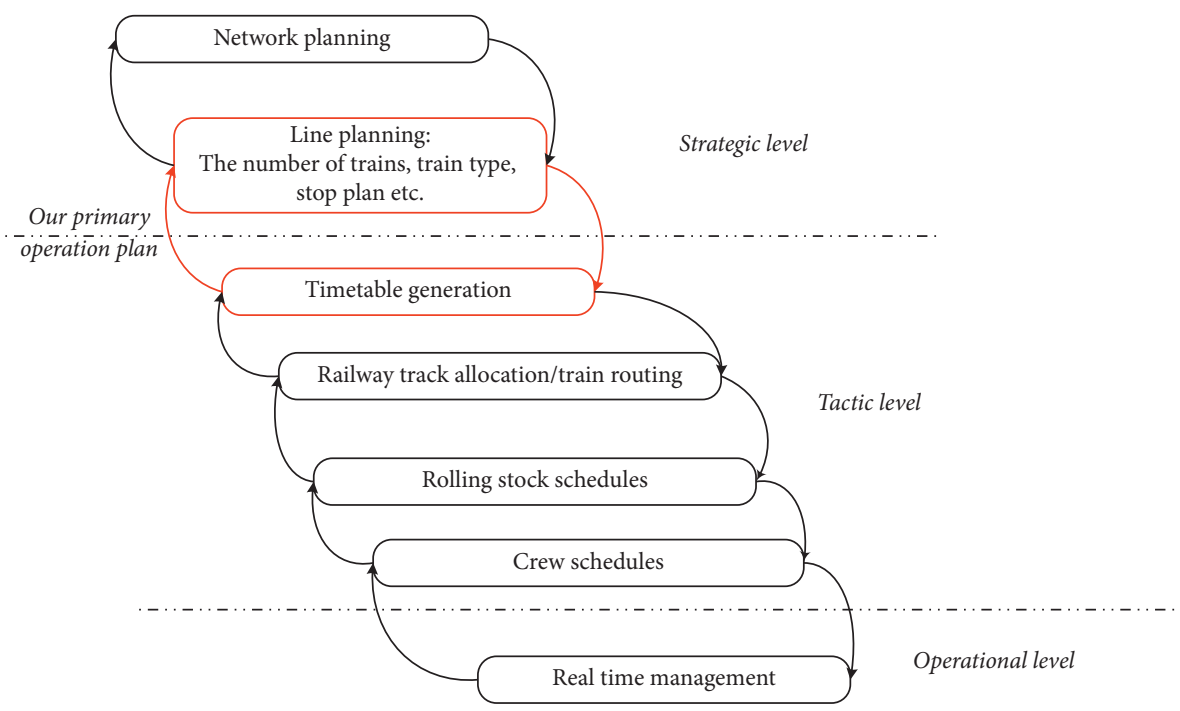

FIgURE 1: The process of designing an operation plan for trains.

paper to obtain the primary operation plan including the number of trains, type of train, stop plan, and timetable. Yang et al. [8] first proposed the collaborative optimization framework for both stop planning and timetable problems. In the previous research, the timetable needs to be regenerated to meet the prespecified stop plan constraints. In Yang et al. [8], the decision variables of the stop plan and timetable are optimized jointly in a model, which reduces the complexity of the problem. This paper further extends the collaborative optimization method in Yang et al. [8] to the problem of adding additional trains, wherein the variables of the number of passengers, stop plan, train type, and timetable are jointly optimized.

Although the specialized operation plan for additional trains is not studied widely, LPP and Train Timetable Problem are two related topics. LPP and TTP are studied separately and two popular problems. LPP is to specify the number of trains, the type of trains, and the stop plan for each train. Two main conflicting objectives exist in the existing optimization models: maximizing the benefits of passengers and minimizing the operational cost of the rail system [2]. In general, there are three types of models proposed in the literature about LPP, namely, (1) cost-responsible models, (2) passenger responsible models, and (3) cost and passenger responsible models. Cost-responsible models are established for the purpose of minimizing the operational cost [9-12]. Passenger responsible models for LPP focus on maximizing the level of service for passengers, that is, the number of direct travelers, travel times, and waiting times of all passengers [13-15]. Cost and passenger responsible models give a trade-off between the cost of the railway company and the satisfaction of passengers $[8,16-18]$. Passenger demand is always ignored in literatures of adding trains. However, it is the main purpose of adding trains that is to meet passenger demand. To balance the benefits of the railway company and the satisfaction of passenger demand, this paper constructs a biobjective model to design a cost and passenger responsible operation plan for additional trains.
The stop planning problem is a subproblem of LPP. Stop plan is to determine stations at which trains stop. The simplest stop plan patterns is all-stop. In the all-stop patterns, trains will stop at all stations along the railway corridor and pick up all passengers at the station. The all-stop pattern can meet all passenger demand but enhance the total travel times and running distance, which does not benefit service for passengers and operational costs. To balance passenger demand, the service for passengers, and operational cost, skip-stop patters are widely applied in practice. Trains may skip the station with low demand to reduce total traveling times and operational cost. Lee et al. $[19,20]$ presented an optimization model under the skip-stop pattern and designed an efficient genetic algorithm to obtain a skip-stop strategy. Niu et al. [21] adjusted the train timetable for a rail corridor under a predetermined skip-stop pattern to minimize the total passenger waiting time at stations. To tradeoff passenger demand and operational cost, this paper studied the problem of adding additional trains also adopting the skip-stop patterns.

The study on TTP is generally to provide an optimal timetable, which both benefits rail company and passengers, for a specific number of trains on a certain rail corridor or network. More and more literatures studied demand-oriented TTP [22-25]. Two types of models to describe the TTP: (1) Mixed-integer linear programming models (MILP) and (2) Integer programming models (IP). Mixed-integer linear programming models are used to solve TTP $[6,24]$. In the mixed-integer linear model, the variables of time are represented by continuous variables. Meanwhile, many disjunctive constraints are introduced to describe the relationship between continuous variables and integer variables. For example, the relationships between variables of departure or arrival times and the variables of stop plan are described by the disjunctive constraints. With the scale of cases increasing, the number of disjunctive constraints is increasing more and more. As we know, too many disjunctive constraints will weaken the solution process. The other type 
of model is the integer programming model in time-space networks. Space-time networks can represent well the spatial and temporal characteristics of railway systems. The problem of railway systems can be transformed into the routing problem in space-time network. The IP models based on the space-time representation method are used in some literatures of TTP $[5,26,27]$. This paper introduces the IP model based on a space-time network to solve the problem of designing an operation plan for additional trains.

1.3. Contributions. This paper makes three main contributions: First, the traditional process of designing an operation plan was simplified into a new process, as illustrated in Figure 1. This process is suitable for designing an operation plan for additional trains because the operation plan should be established in a short time to quickly provide suitable transport capacity for the passenger demand in peak periods. The joint optimization framework is used to determine the number of additional trains, the stopping plan, the train types, and the timetable simultaneously. However, due to the many variables, the complexity of the construction model is high. Thus, using the space-time network representation, we transform the problem of designing the primary operation plan into a multiple-train path planning problem in a space-time network. By finding a set of feasible paths for additional trains, a feasible primary operation plan for additional trains is obtained.

Second, we present a new biobjective integer programming model for the design of the primary operation plan for additional trains on a long-trip high-speed rail corridor. Two conflicting objectives, namely, minimization of the total deviation between the provided transport capacity and the passenger demand and minimization of the total travel times of additional trains, are introduced into the model. Via the formation of the objective function of passenger demand and the loading capacity constraint, we can generate the stopping plan successfully for additional trains to provide a loading capacity that is close to the passenger demand of each station. According to the objective function of the total travel times, trains skip stations with small passenger demand. The integration of the two objective functions via linear weighting yields a trade-off between the passenger demand and the travel times. The number of additional trains is also determined according to the weights of the two objectives. In addition, the attendance rate constraints guarantee that the stopping plan for each additional train can provide not only sufficient loading capacity but also satisfactory service for passengers. The train type constraint can maintain a specified proportion of various types of trains to provide more choices for passengers. Safety headway constraints are used to ensure that the additional trains run safely on the rail corridor and do not disturb the operation of the original trains for the safety and stability of the whole rail system.

Third, two sets of experiments are conducted on a ninestation rail corridor and Wuhan-Guangzhou high-speed rail corridor to evaluate the effectiveness and efficiency of our proposed methods. We use the CPLEX solver to solve the proposed model. The results on the small-scale example demonstrate the satisfactory performance of the proposed methods. Furthermore, via the adoption of various sets of parameters and strategies, we evaluate the results of the large-scale experiments. The experimental results demonstrate that our proposed methods can generate an optimal primary operation plan within acceptable computation times. The selected parameters and strategies affect the results of the experiments. Operators can select the parameters according to the application requirements.

The remainder of this paper is organized as follows: In Section 2, we provide a detailed problem statement. In Section 3, we present the assumptions and notations of the considered problem. Then, a biobjective integer programming model for the design of the primary operation plan is formulated. The complexity of the model is also discussed in this section. In Section 4, we conduct two sets of experiments to evaluate the performance of the proposed approach. In Section 5, the conclusions of this study are presented and future work is discussed.

\section{Problem Statement}

In this paper, a double-track railway corridor is considered as the physical environment of the problem. The railway corridor consists of stations that are indexed by $i \in S\{1,2, \ldots, S\}$, and adjacent stations are connected by a segment, which includes double tracks; see Figure 2. Moreover, in this double-track railway corridor model, trains that travel in the inbound and outbound directions are mutually independent. Without loss of generality, in this study, only the scheduling of additional trains in the inbound segments is considered.

The set of trains that are considered in the model is $K_{1} \cup K_{2}$, where $K_{1}$ denotes the set of original trains in the offpeak period and $K_{2}$ denotes the set of additional trains that the operators expect to add during the peak period. In the off-peak period, only the original trains travel on the railway corridor, while in the peak period, the operators must add additional trains to improve the transportation capacity and to satisfy the passenger demand. The safe and efficient operations of additional trains significantly impact passengers' trips during the peak period. In this paper, we study the problem of designing a primary operation plan that includes the number of additional trains, the train types, the stopping plan, and the timetable for the additional trains.

As above, we aim to obtain the primary operation plan on the tactic levels. Thus, we here only consider the macro demands at each station instead of counting the number of passengers boarding and alighting. The number of passengers at each station can be approximately obtained through historical passenger demand data. Obviously, the macro demand can cause the loss of accuracy. Thus, our operation plan in this paper is just a primary operation plan which needs to be adjusted and reoptimized in the next other stages.

The number of additional trains should meet the passenger demand during the peak period as much as possible but not be so large as to cause a waste of transport 


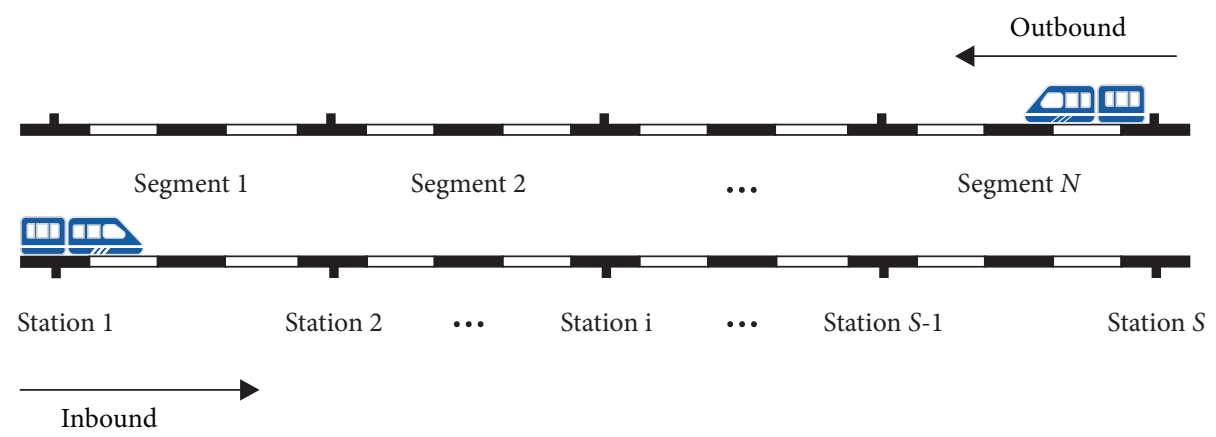

FIGURE 2: A high-speed railway corridor with double tracks.

capacity. Additionally, since the departure and arrival times of an additional train must maintain moderate time intervals, namely, arrival headway and departure headway, with its adjacent trains to ensure the safety of operation, only finitely many trains can be added within a limited time horizon.

In this paper, we consider the China high-speed railway corridor as the decision-making environment, and all the trains in this study are categorized into two types (denoted by $G$ and $D$ ) according to their maximum allowed speeds. Here, $G$ and $D$ represent types of trains with maximum velocities of $350 \mathrm{~km} / \mathrm{h}$ and $250 \mathrm{~km} / \mathrm{h}$, respectively. Since (1) there are only finitely many $G$-trains and (2) the cost and ticket price of $D$-trains are lower, a specified number of $D$-trains should always be present in the additional train plan.

The stopping plan specifies whether a train stops or not at a station according to the predicted passenger demand. The stopping plan affects the quality of service for passengers and the trains' operation efficiency. Thus, we should establish a trade-off between maximizing the travel convenience of passengers and minimizing the operation cost when we design the stopping plan for additional trains.

The timetable determines the arrival, departure, and dwelling times of each additional train at each station. In this study, the timetable of the original trains is fixed. Thus, the timetable for the additional trains must satisfy a headway constraint to ensure that the additional trains do not disturb the operation of the original trains.

Based on the spatial and temporal characteristics of the operation plan, the design of the primary operation plan for trains can be generalized to a space-time decision-making problem. A space-time network considers both physical paths and the time horizon, and it is a powerful tool for our problem. For convenience, the problem is treated with two processes: (1) the rail corridor is simplified as a physical path graph $G_{1}(V, E)$, in which stations are represented by nodes in $V$ and inbound/outbound segments are represented by arcs in $E$, and (2) the time horizon is separated into a set of timestamps, which is denoted by $T=\{0, \delta, 2 \delta, \ldots, N \delta\}$, where $\delta$ is assumed to be an interval of time length $\delta$ and $N$ is a positive integer that is sufficiently large for ensuring that the interval $[0, N \delta]$ covers the planning time horizon. With the timestamps of the time horizon, the physical path graph $G_{1}(V, E)$ is extended into a space-time network graph $G_{2}\left(S, S^{\prime}, A, A^{\prime}\right)$, in which $S$ and $A$ represent the sets of space-time nodes and space-time travel arcs that correspond to $V$ and $E$, respectively, in the physical network. In addition, dummy space-time nodes $S^{\prime}$ that are related to $V$ and station arcs $A^{\prime}$ are added into the space-time network.

A small-scale space-time network with the trajectory of one train is illustrated in Figure 3. We consider a physical path that consists of three segments from node 1 to node 4 , which represents a rail corridor from station 1 (origin) to station 4 (destination). The time horizon is separated into 21 timestamps, namely, $\{0, \delta, 2 \delta, \ldots, 21 \delta\}$, which are embedded into the physical path for the construction of a twodimensional network that has both spatial and temporal characteristics. Dummy space-time nodes that are related to each physical node at each timestamp are also added into the space-time network to represent the stopping plan. Each physical node of the physical path is associated with two space-time nodes at each timestamp. Travel arcs, which connect two space-time nodes of adjacent physical nodes, are considered as optional paths for the specification of the departure times, the arrival times, the link travel times, and the types of trains. In this paper, two types of travel arcs are defined according to the travel times of the two types of trains on each segment. For example, the travel arcs with shorter travel times represent optional paths of $G$-trains, and those with longer travel times represent optional paths of $D$-trains. In addition, two types of station arcs, which connect two space-time nodes of the same physical node, namely, stop and nonstop arcs, are defined to represent the stopping plans of trains, where the stop arcs are used to describe the stopping plans and the dwelling times of trains at the station. For example, in Figure 3, a space-time trajectory of a train is presented to illustrate the operating process. A $D$-train departs from station 1 (origin) at timestamp $5 \delta$ and arrives at station 2 at timestamp $8 \delta$. Then, the train does not stop at station 2 and departs from station 2 at timestamp $8 \delta$. Next, the train arrives at station 3 at timestamp $10 \delta$ and dwells for a time interval at station 3 . After that, the train departs from station 3 at timestamp $11 \delta$ and arrives at station 4 (destination) at timestamp $14 \delta$.

Based on the space-time network in Figure 3, an example is presented as Figure 4 to illustrate the process of 


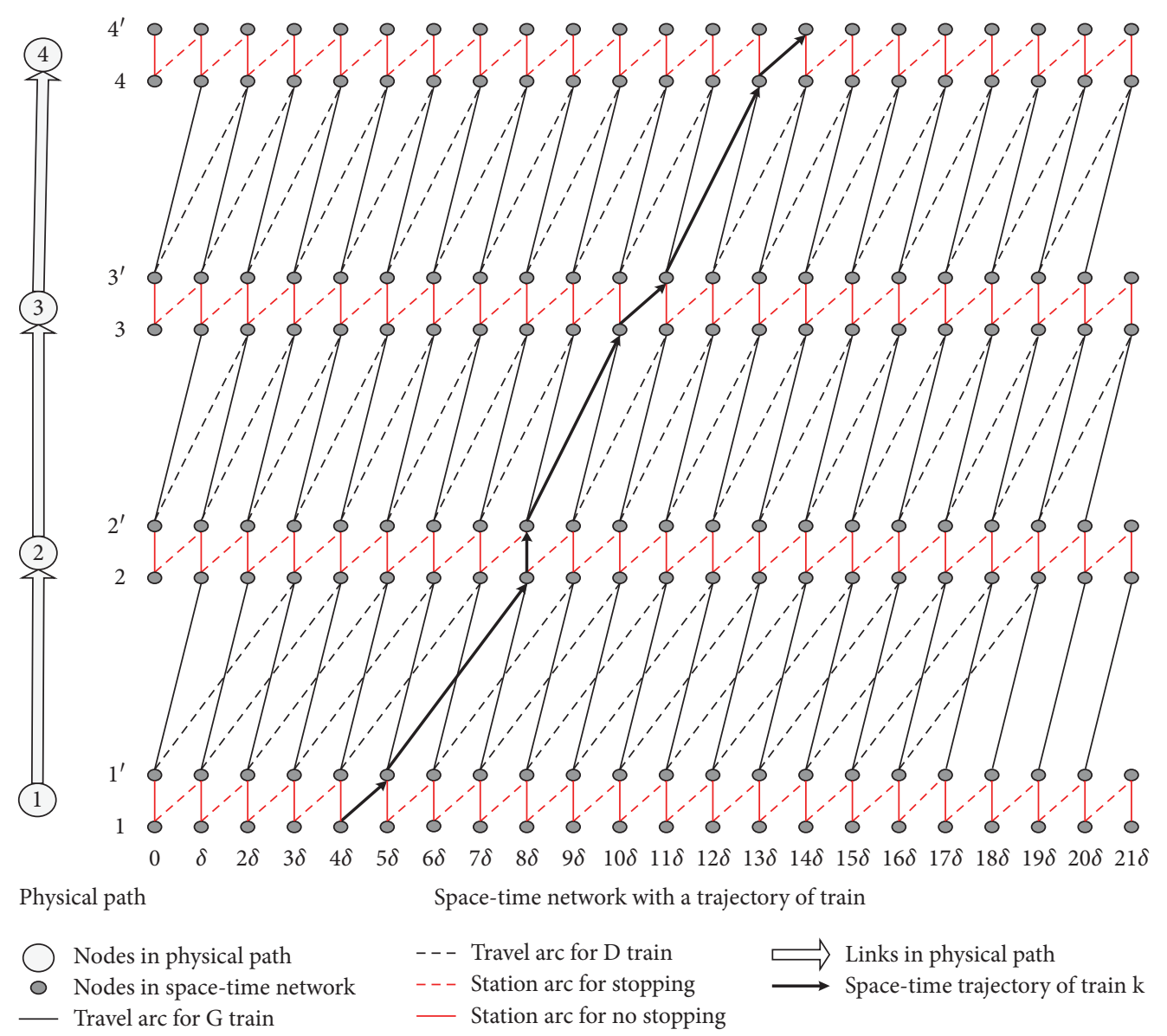

Figure 3: The space-time path of a train in the space-time network.

designing an operation plan for additional trains in the peak period. As shown in Figure 4, there are three original trains $(T 1, T 2, T 3)$, of which the origin station is station 1 and the destination is station 4 . In an attempt to satisfy the passenger demand in the peak period, two additional trains, namely, $T 4$ ( $G$-train) and $T 5$ ( $D$-train), are added. In Figure 4, the black lines and red lines represent the space-time paths of the original and additional trains, respectively. According to the trajectories of the additional trains, various train types, stopping plans, and departure and arrival times are selected according to the operation requirements by finding paths in the space-time network. For example, $T 4$ and $T 5$ are designed as different train types because they select different types of travel arcs as paths. In addition, as the passenger demand differs among the stations, both $T 4$ and $T 5$ are scheduled to stop at station 2, while only $T 5$ is scheduled to stop at station 3. According to this example, the problem of designing an operation plan for additional trains can be transformed into a multiple-train path planning problem in a spacetime network. Furthermore, if binary decision variables are introduced as indicators of whether the space-time paths are selected or not, the feasible solutions can be obtained to represent the temporary plan for additional trains; namely, we can model this problem as a $0-1$ integer programming model.

\section{Mathematical Formulation}

In this section, a mathematical model is constructed for obtaining the optimal operation plan for additional trains. First, several assumptions are made to simplify the problem.

Assumption 1. Only long-trip trains are considered. All trains depart from station 1 and finally reach station $S$.

Assumption 2. The travel times of trains of the same train type along a segment are fixed, and the dwelling times of trains at a station are fixed. In this study, the travel times of trains are only related to the train type and the segment, and the dwelling times of trains depend on the level of the station.

Assumption 3. Overtaking operations are not permitted under any circumstances; that is, it is forbidden no matter what at stations or within segments.

Assumption 4. Empirical estimates of the loading capacity of each train and the passenger demand at each station are used in this study. The number of passengers who get on and off each train at each station are not investigated in this study. 


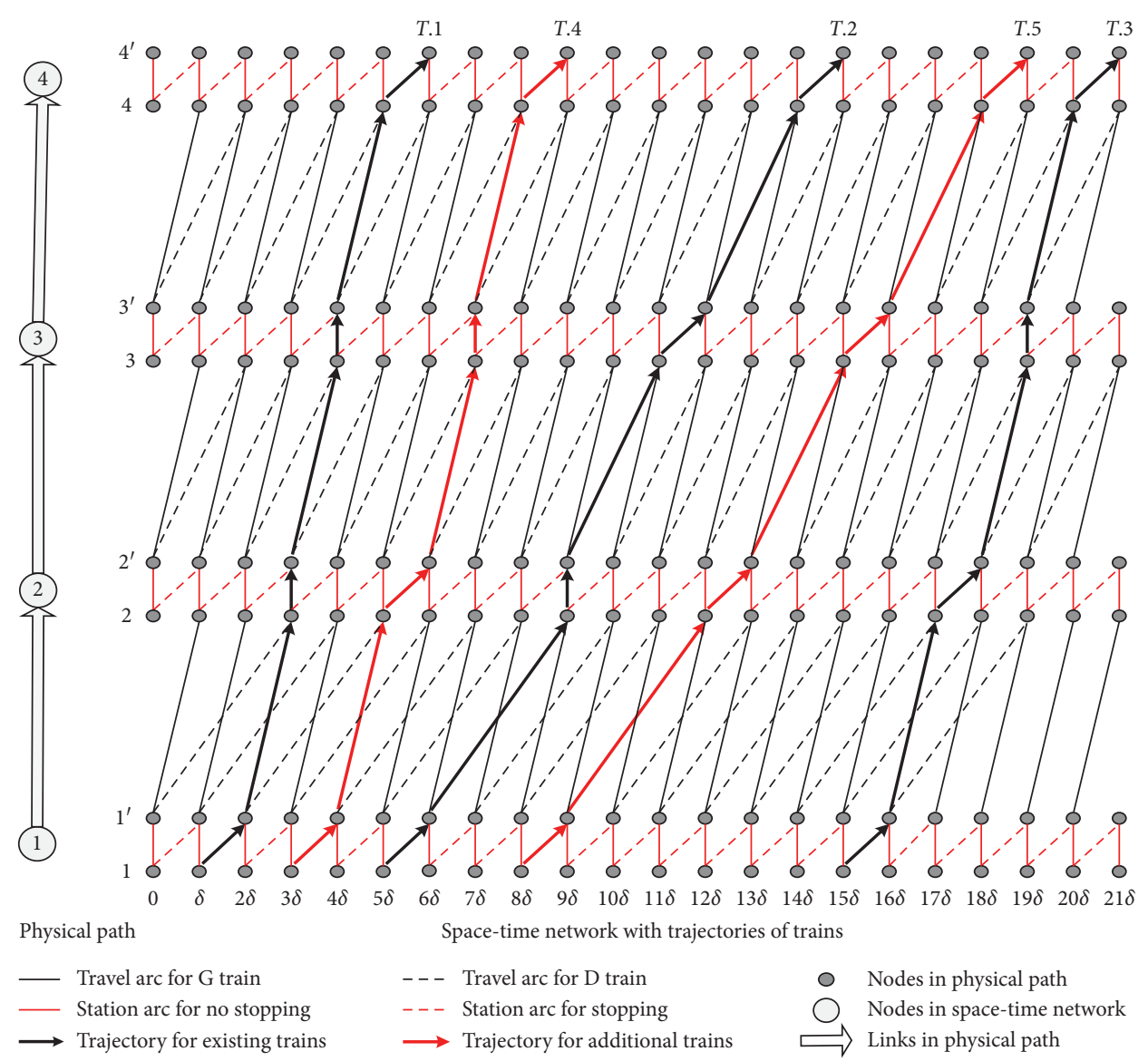

FIGURE 4: An illustration of adding trains on the space-time network.

Assumption 5. The capacity of the station and the rolling stocks are unlimited.

3.1. Notations and Parameters. For the reader's convenience, all the notations and parameters that are used in the study are defined in Table 1.

3.2. Decision Variables. To generate a feasible operation plan, we must specify the space-time trajectory for each train. Thus, the problem is transformed into an optimal path choice process for multiple trains in a space-time network, which involves three binary decision variables:

$x_{i^{\prime}, i+1, t, l}^{k}$ : selection indicator of the additional train $k$ for space-time travel arc $\left(i^{\prime}, i+1, t, l\right)$, which equals 1 if the train $k$ of type $l$ enters segment $(i, i+1)$ at time $t$ and equals 0 otherwise;

$x_{i, i^{\prime}, t, p}^{k}$ : selection indicator of the additional train $k$ for space-time station arc $\left(i, i^{\prime}, t, p\right)$, which equals 1 if train $k$ chooses stopping plan $p$ at station $i$ at time $t$ and equals 0 otherwise;

$y^{k}$ : selection indicator of the additional train $k$ being added, which is also a binary variable. It equals 1 if additional train $k$ satisfies the condition $\sum_{\left(i^{\prime}, i+1, t, l\right) \in A^{\prime}} x_{i^{\prime}, i+1, t, l}^{k}+\sum_{\left(i, i^{\prime}, t, p\right) \in A^{\prime}} x_{i, i^{\prime}, t, p}^{k}>0$ and equals 0 otherwise

$N_{D}$ : number of $D$-trains;

$N_{A}$ : number of additional trains.

Variables $x_{i^{\prime}, i+1, t, l}^{k}$ and $x_{i, i^{\prime}, t, p}^{k}$ are typically associated with the generation of the feasible space-time path, which represents the feasible operation plan, for each train $k \in K_{2}$, where $K_{2}$ denotes a set of trains: $x_{i^{\prime}, i+1, t, l}^{k}$ determines the departure time and the train type of train $k \in K_{2}$, while $x_{i, i^{\prime}, t, p}^{k}$ determines the stopping plan of train $k \in K_{2}$.

In addition, $K_{2}=\left\{1,2, \ldots,\left|K_{2}\right|\right\}$ denotes the set of additional trains that operators expect to add, where $\left|K_{2}\right|$ is the maximum number of additional trains, to provide the origin with the sufficient loading capacity, and it is defined as follows:

$$
\left|K_{2}\right|=\frac{Q_{1}-\sum_{k \in K_{1}} \sum_{t \in T} \sum_{p \in P} q_{i}^{k} a_{1,1^{\prime}, t, p}^{k}}{q^{1}}
$$

Not all trains in $K_{2}$ are added into the operation plan in the peak period under practical conditions. Therefore, we introduce a state variable $y^{k}$ into the model according to whether the train $k \in K_{2}$ is added in the optimal solution or not, and the number of additional trains can be calculated as follows: 
TABLE 1: Notations and parameters in this problem.

\begin{tabular}{|c|c|}
\hline Notation & Definition \\
\hline$K_{1}=\left\{1,2, \ldots,\left|K_{1}\right|\right\}$ & Set of original trains \\
\hline$K_{2}=\left\{1,2, \ldots,\left|K_{2}\right|\right\}$ & Set of additional trains that operators expect to add \\
\hline$G$ & Set of $G$-trains \\
\hline$D$ & Set of $D$-trains \\
\hline$T=\{1,2, \ldots,|T|\}$ & Set of timestamps in the time horizon \\
\hline$V=\{1,2, \ldots,|V|\}$ & Set of physical nodes, which represent all stations \\
\hline$V^{\prime}=\left\{1,2, \ldots,\left|V^{\prime}\right|\right\}$ & Set of dummy physical nodes, which represent all stations \\
\hline$E=\{1,2, \ldots,|E|\}$ & Set of physical links, which represent segments \\
\hline$L=\{1,2\}$ & Set of train types: 1 indicates $G$-train and 2 indicates $D$-train \\
\hline$P=\{1,2\}$ & Set of stopping plans: 1 indicates nonstop and 2 indicates stop \\
\hline$S$ & Set of space-time nodes \\
\hline$S^{\prime}$ & Set of dummy space-time nodes \\
\hline$A$ & Set of space-time travel arcs \\
\hline$A^{\prime}$ & Set of space-time station arcs \\
\hline$k$ & Index of trains, $k \in K_{1} \cup K_{2}$ \\
\hline$t$ & Time index, $t \in T$ \\
\hline$i, j$ & Index of physical nodes, $i, j \in V$ \\
\hline$i^{\prime}, j^{\prime}$ & Index of dummy physical nodes that correspond to $i, j, i^{\prime}, j^{\prime} \in V^{\prime}$ \\
\hline$l$ & Index of train types, $l \in L$ \\
\hline$p$ & Index of stopping plans, $p \in P$ \\
\hline$(i, t)$ & Index of space-time travel nodes, $(i, t) \in S$ \\
\hline$\left(i^{\prime}, t\right)$ & Index of dummy space-time travel nodes, $\left(i^{\prime}, t\right) \in S^{\prime}$ \\
\hline$(i, i+1)$ & Index of physical links that represent segments, $(i, i+1) \in E$ \\
\hline$\left(i^{\prime}, i+1, t, l\right)$ & Index of space-time travel arcs, $\left(i^{\prime}, i+1, t, l\right) \in A$ \\
\hline$\left(i, i^{\prime}, t, p\right)$ & Index of space-time station arcs, $\left(i, i^{\prime}, t, p\right) \in A^{\prime}$ \\
\hline$t_{i, i+1}^{l}$ & Travel times of trains of type $l$ on segment $(i, i+1)$ \\
\hline$d_{i}^{p}$ & Dwell time of trains on station $i$ in stopping plan $p$ \\
\hline$a_{i^{\prime}, i+1, t, l}^{k}$ & Selection indicator of the original train $k$ for space-time travel arc $i^{\prime}, i+1, t, l$ \\
\hline$a_{i, i^{\prime}, t, p}^{k}$ & Selection indicator of the original train $k$ for space-time station $\operatorname{arc}\left(i, i^{\prime}, t, p\right)$ \\
\hline$q_{i}^{k, l, t, p}$ & Loading capacity of train $k$ at station $i$ \\
\hline$Q_{i}$ & Passenger demand at station $i$ \\
\hline$Q$ & Total passenger demand of all stations \\
\hline$h_{\mathrm{dmin}}$ & Minimal departure headway \\
\hline$h_{\text {amin }}$ & Minimal arrival headway \\
\hline$N_{\min }^{D}$ & Required minimal number of $D$-trains \\
\hline$\mu_{k}$ & Attendance rate of train $k$ \\
\hline$\mu_{\max }$ & Maximum attendance rate \\
\hline$\mu_{\min }$ & Minimal attendance rate \\
\hline$C$ & Capacity of each train \\
\hline
\end{tabular}

$$
N_{A}=\sum_{k \in K_{2}} y^{k}
$$

3.3. Formulation of the Constraints. Four sets of constraints are considered in the process of designing the operation plan: (i) unique space-time path constraints; (ii) safety headway constraints; (iii) train type constraints; and (iv) loading capacity constraints. Detailed formulations of each set of constraints are presented in the following parts.

3.3.1. Unique Space-Time Path Constraints. To guarantee that at most one connecting path is generated from the origin to the destination for each train $k \in K_{2}$ in the spacetime network, a series of space-time path constraints are presented. We require that if the train $k \in K_{2}$ is added in the peak period, up to one travel arc is selected that corresponds to each physical link for each train $k \in K_{2}$. Similarly, at most, one station arc will be chosen that corresponds to each station for each train $k \in K_{2}$. Thus, constraints (3) and (4) are defined as follows:

$$
\begin{gathered}
\sum_{t \in T} \sum_{l \in L} x_{i^{\prime}, i+1, t, l}^{k}=y^{k}, \quad \forall k \in K_{2} ; \forall i \in V /\{|V|\}, \\
\sum_{t \in T} \sum_{p \in P} x_{i, i^{\prime}, t, p}^{k}=y^{k}, \quad \forall k \in K_{2} ; \forall i \in V .
\end{gathered}
$$

Furthermore, to ensure that all the selected travel arcs and station arcs of each train $k$ can constitute a connecting path from the origin to the destination in the space-time network, we balance the incoming travel arc and the outgoing station arc for each space-time node $(i, t) \in S /\{(1, t)\}$ and the incoming station arc and the outgoing travel arc for each dummy space-time node $\left(i^{\prime}, t\right) \in S^{\prime} /\left\{\left(\left|V^{\prime}\right|, t\right)\right\}:$ 


$$
\begin{aligned}
& \left\{\begin{array}{l}
\sum_{l \in L} x_{i^{\prime}, i+1, t, l}^{k}=0, \quad \forall k \in K_{2} ; i=|V| ; \forall t \in T, \\
\sum_{l \in L} x_{i^{\prime}, i+1, t, l}^{k}-\sum_{p \in P} x_{i, i^{\prime}, t-d_{i}^{p}, p}^{k}=0, \quad \forall k \in K_{2} ; \forall i \in \frac{V}{\{|V|\}} ; \forall t \in T \text { and } t-d_{i,}^{p},
\end{array}\right. \\
& \sum_{l \in L} x_{i^{\prime}, i+1, t, l}^{k}=0, \quad \forall k \in K_{2} ; \forall i \in \frac{V}{\{|V|\}} ; \forall t \in T \text { and } t-d_{i}^{p} \notin T, \\
& \left\{\begin{array}{l}
\sum_{p \in P} x_{i, i^{\prime}, t, p}^{k} \leq 1, \quad \forall k \in K_{2} ; i=1 ; \forall t \in T, \\
\sum_{p \in P} x_{i, i^{\prime}, t, p}^{k}-\sum_{l \in L} x_{(i-1)^{\prime}, i, t-t_{i, i+1}^{l}, l}^{k}=0, \quad \forall k \in K_{2} ; \forall i \in \frac{V}{\{1\}} ; \forall t \in T \text { and }, t-t_{i, i+1}^{l} \in T, \\
\sum_{p \in P} x_{i, i^{\prime}, t, p}^{k}=0, \quad \forall k \in K_{2} ; \forall i \in \frac{V}{\{1\}} ; \forall t \in T \text { and }, t-t_{i, i+1}^{l} \notin T .
\end{array}\right.
\end{aligned}
$$

3.3.2. Safety Headway Constraints. To ensure safe operation, additional trains must be scheduled under operational restrictions on the departure and arrival times to avoid collisions. Since in the space-time network the departure and arrival times of trains are represented by space-time arcs, the operation plans of additional trains can be controlled by implementing restrictions on the selection of the space-time paths. Thus, additional trains must avoid selecting incompatible arcs as paths. Incompatible arcs are travel arcs that have been already selected as paths by other trains; the selection of such an arc could cause a collision. As a result, in this model, the sum of all incompatible arcs should be less than 1 . These constraints are expressed in detail as follows:

(1) Departure and Arrival Headway Constraints. For the security of interstation operations, if two consecutive trains depart from or arrive at a station, we should set a time interval between the departure/arrival times of these two trains in preparation for each train's arrival or departure operations. In this study, the departure and arrival time intervals are called the departure and arrival headways. Then, for each added train, the departure and arrival headways should not only be considered in the arrival/departure operations of other adjacent additional trains but also be applied to the original trains.

An implementation case of the departure headway constraint is illustrated in Figure 5. For two consecutive trains that depart from station $i$ in succession, if the first train departs at time $t_{0}$, to satisfy the specified headway constraint, the second train should not depart from station $i$ earlier than $t_{0}+h_{d \text { min }}$, where $h_{d \text { min }}$ is the minimal departure headway.

For example, in Figure 5, for the travel arc that is associated with space-time node $\left(i^{\prime}, t_{0}\right)$, the next compatible arc must be the travel arc that is associated with space-time node $\left(i^{\prime}, t_{0}+h_{d \text { min }}\right)$; namely, all travel arcs that are related to the space-time nodes within the range of $\left\{\left(i^{\prime}, t_{0}\right), \ldots,\left(i^{\prime}, t_{0}+h_{d \min }-\delta\right)\right\}$ are incompatible, where $\delta$ is a unit time interval. As a result, the sum of the decision variables and other parameters of the original trains that are involved with these incompatible arcs should be less than 1. The departure headway constraint is expressed as follows:

$$
\begin{gathered}
\sum_{k \in K_{2}} \sum_{l \in L} \sum_{t \in\left\{t_{0}, \ldots, t_{0}+h_{d \min }-1\right\}} x_{i^{\prime}, i+1, t, l}^{k}+\sum_{k \in K_{1}} \sum_{l \in L} \sum_{t \in\left\{t_{0}, \ldots, t_{0}+h_{d \min }-1\right\}} a_{i^{\prime}, i+1, t, l}^{k} \leq 1, \\
\forall i \in V /\{|V|\} ; \forall t_{0} \in T \text {, and } t_{0}+h_{d \min }-1 \in T .
\end{gathered}
$$

Similarly, an implementation case of the arrival headway constraint is illustrated in Figure 6. If the station arc that is associated with space-time node $\left(i, t_{0}\right)$ is selected as the path for the first train, which arrives at station $i$ at time $t_{0}$, then the next train must select the station arc that is associated with space-time node $\left(i, t_{0}+h_{d \text { min }}\right)$ as its path to avoid conflict; namely, the station arcs that are related to the space-time nodes within $\left\{\left(i, t_{0}\right), \ldots,\left(i, t_{0}+h_{d \text { min }}-\delta\right)\right\}$ are incompatible. The arrival headway constraint on the incompatible arcs is expressed as follows:

$$
\begin{gathered}
\sum_{k \in K_{2}} \sum_{p \in P} \sum_{t_{0}, \ldots, t_{0}+h_{a \min }-1} x_{i, i^{\prime}, t, p}^{k}+\sum_{k \in K_{1}} \sum_{p \in P} \sum_{t \in\left\{t_{0}, \ldots, t_{0}+h_{a \min }-1\right\}} a_{i, i^{\prime}, t, p}^{k} \leq 1, \\
\forall i \in V /\{1\} ; \forall t_{0} \in T, \text { and } t_{0}+h_{a \min }-1 \in T .
\end{gathered}
$$

(2) Tracking Headway Constraint. The tracking headway constraint is applied to avoid collision between two consecutive tracking trains that are traveling on the same segment. There are three types of tracking operation: two trains of the same type; a $D$-train goes after a $G$-train; and a $G$-train goes after a $D$-train. Based on the assumption that trains of the same type share the same velocity and travel times, trains of the first type of tracking operation can operate safely under departure and arrival headway constraints. In addition, since a $G$-train is much faster than a 


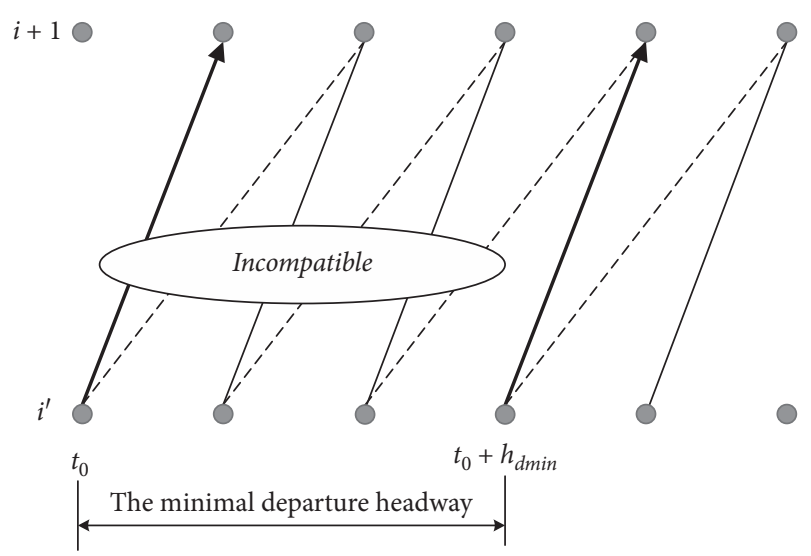

Figure 5: An illustration for departure headway constraint.

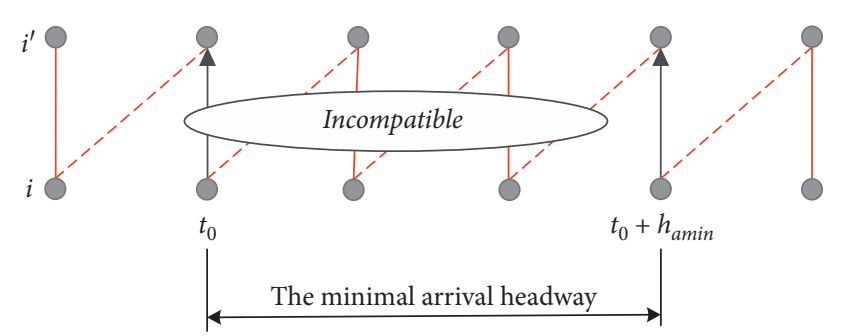

Figure 6: An illustration for arrival headway constraint.

$D$-train, collisions never occur in the second type of operation. However, two consecutive trains in the third type of operation are at risk of collision; see Figure 7.

An example of the third type of tracking operation is illustrated in Figure 7, where $t_{i, i+1}^{2}-t_{i, i+1}^{1}$ denotes the difference between the $G$-train's travel time and the $D$-train's travel time on the segment $(i, i+1)$. For this segment, if $t_{i, i+1}^{2}-t_{i, i+1}^{1}>\delta$, collisions may occur between these two consecutive trains: If the $D$-train departs from station $i$ at time $t_{0}$, and the following $G$-train departs from station $i$ at time $t_{0}+\delta$ or $t_{0}+2 \delta$, train collision would result. To avoid a collision, the following train should depart from station $i$ no earlier than $t_{0}+t_{i, i+1}^{2}-t_{i, i+1}^{1}$. Hence, the $\operatorname{arc}\left(i^{\prime}, i+1, t_{0}, 2\right)$ is incompatible with other arcs that are associated with spacenodes within $\left\{t_{0}+1, \ldots, t_{0}+t_{i, i+1}^{2}-t_{i, i+1}^{1}-\delta\right\}$. The decision variables of the additional trains and the parameters of the original trains that are involved with these incompatible arcs should satisfy the following condition:

$$
\begin{aligned}
& \sum_{k \in K_{2}}\left(x_{i^{\prime}, i+1, t_{0}, 2}^{k}+\sum_{t \in\left\{t_{0}+1, \ldots, t_{0}+t_{i, i+1}^{2}-t_{i, i+1}^{1}-1\right\}} x_{i^{\prime}, i+1, t, 1}^{k}\right) \\
& +\sum_{k \in K_{1}}\left(a_{i^{\prime}, i+1, t_{0}, 2}^{k}+\sum_{t \in\left\{t_{0}+1, \ldots, t_{0}+t_{i, i+1}^{2}-t_{i, i+1}^{1}-1\right\}} a_{i^{\prime}, i+1, t, 1}^{k}\right) \leq 1, \\
& \quad \forall i \in V /\{|V|\} \text { and } t_{i, i+1}^{2}-t_{i, i+1}^{1}>1 ; \forall t_{0} \in T \text { and } t_{0}+t_{i, i+1}^{2} \\
& -t_{i, i+1}^{1}-1 \in T .
\end{aligned}
$$

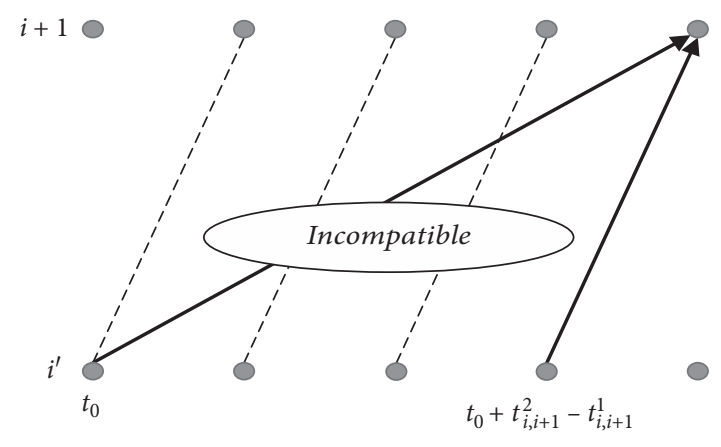

FIGURE 7: An illustration for tracking headway constraint.

3.3.3. Train Type Constraints. In practice, once a train has been determined to run on a specified rail corridor from origin to destination, its train type never changes. In this paper, a train type constraint is applied to ensure that on all travel arcs that are chosen as space-time paths for each additional train $k \in K_{2}$, the train type remains the same:

$$
\sum_{t \in T} x_{i^{\prime}, i+1, t, l}^{k}=\sum_{t \in T} x_{j^{\prime}, j+1, t, l}^{k}, \quad \forall k \in K_{2} ; \forall i, j \in \frac{V}{\{|V|\}} ; \forall l \in L .
$$

Although the $D$-train is slower than the $G$-train, the ticket price of the $D$-train is lower. To provide more choices for passengers, we impose a train type constraint that ensures that at least a specified number of $D$-trains are involved in the additional train plan:

$$
\sum_{k \in K_{2}} \sum_{t \in T} x_{i^{\prime}, i+1, t, 2}^{k} \geq N_{\min }^{D}, \quad \forall i \in V
$$

where $N_{\min }^{D}$ is the threshold value of the required minimum number of $D$-trains.

\subsubsection{Loading Capacity Constraints}

(1) Constraint on the Loading Capacity for Each Station. The objective of this paper is to provide sufficient loading capacity for the satisfaction of the increasing passenger demand during the peak period. In this paper, only long-trip trains on the rail corridor are considered, which not only can provide abundant loading capacity to satisfy most of the passenger demand but also avoid waste of transportation resources. Passengers whose demand is not satisfied by this operation plan can commute between their origin and destination by taking other short-trip trains and by transferring several times.

However, since the track capacity of the rail corridor and the number of additional trains that can be added into the operation plan are both finite, it is possible that the maximum loading capacity that is provided by these long-distance trains will still fail to satisfy the passenger demand of each station in the peak period. Mathematically, the total loading capacity of the additional trains and the original trains that is provided to each station $i \in S$ does not exceed the passenger demand at each station $i \in S$ : 


$$
\sum_{k \in K_{2}} \sum_{t \in T} \sum_{p \in P} q_{i}^{k} x_{i, i^{\prime}, t, p}^{k}+\sum_{k \in K_{1}} \sum_{t \in T} \sum_{p \in P} q_{i}^{k} a_{i, i^{\prime}, t, p}^{k} \leq Q_{i}, \quad \forall i \in V,
$$

where $q_{i}^{k}$ represents the loading capacity of train $k$ at station $i$, which is determined mainly by the type of train, the level of the stations, and empirical historical data on passenger demand, among other factors. $Q_{i}$ is the estimated passenger demand at station $i$, which is obtained based on historical travel data. As specified in Assumption 5, instead of considering the exact numbers of passengers who get on and off each train at each station, the passenger demand at each station is considered from a microscopical perspective.

(2) Attendance Rate Constraints. These constraints are used to restrict the loading capacity of each additional train in the operation plan. The attendance rate is an important factor for measuring the level of utilization of a train's capacity. The attendance rate is calculated by dividing the total passenger load of the train by the maximum capacity of the train. Based on this method, we define the attendance rate of train $k$ as follows:

$$
\mu_{k}=\frac{\sum_{\left(i, i^{\prime}, t, p\right) \in A^{\prime}} q_{i}^{k} x_{i, i^{\prime}, t, p}^{k}}{C}, \quad \forall k \in K_{2} \forall i \in V .
$$

Under an excessive attendance rate, too many passengers are loaded by a train, which leads to low-quality service. Thus, an attendance rate constraint is imposed to limit the attendance rate of the additional train $k \in K_{2}$ to avoid overload of the train:

$$
\mu_{k} \leq \mu_{\max }, \quad \forall k \in K_{2},
$$

where $\mu_{\max }$ denotes the threshold value of the maximum attendance rate of each train.

In contrast, under a low attendance rate, the train's capacity is underutilized. To avoid the waste of the train's capacity, the attendance rate of the added train that is derived from the optimal solution should exceed the minimum attendance rate $\mu_{\min }$. In addition, if the train $k$ is not involved, $\mu_{k}$ will be set to zero, since not all trains in $K_{2}$ will be added in the final optimal solution. Thus, we use the binary variable $y^{k}$ to formulate the model, and these two disjunctive constraints are expressed as follows:

$$
\begin{aligned}
& \mu_{\min }-M y^{k}<\mu_{k}, \quad \forall k \in K_{2}, \\
& \sum_{\left(i^{\prime}, i+1, t, l\right) \in A} x_{i^{\prime}, i+1, t, l}^{k} \leq M\left(1-y^{k}\right), \quad \forall k \in K_{2} \forall i \in V,
\end{aligned}
$$

where $\mu_{\min }$ is the threshold value of the required minimum attendance rate of each train and $M$ is a sufficiently large number. At least one of these two constraints should be satisfied.

3.4. Objective. To improve the quality of service for passengers, operations with shorter travel times and dwelling times are preferred. In addition, to minimize the cost to the railway company, the number of additional trains should be minimized in the operation plan under the condition that as much of the passenger demand be satisfied as possible. Thus, the first objective is to minimize the additional trains' total travel times:

$$
T_{\text {total }}=\sum_{k \in K_{2}} \sum_{\left(i^{\prime}, i+1, t, l\right) \in A} t_{i, i+1}^{l} x_{i^{\prime}, i+1, t, l}^{k}+\sum_{k \in K_{2}} \sum_{\left(i, i^{\prime}, t, p\right) \in A^{\prime}} d_{i}^{p} x_{i, i^{\prime}, t, p}^{k} .
$$

The first term on the right-hand side of equation (18) is the total link travel time, which determines the number of additional trains and the types of the train since the link travel times are the same for each train type. The second term is the total train dwelling time, which is related to the stopping plan of the additional trains.

To satisfy as much passenger demand as possible in the peak period, the difference between the total passenger demand and the total supply capacity of all trains, namely, both the original trains and the additional trains, should be minimized. Thus, the second objective function minimizes the difference:

$$
Q_{d}=Q-\sum_{k \in K_{2}} \sum_{\left(i, i^{\prime}, t, p\right) \in A^{\prime}} q^{i} x_{i, i^{\prime}, t, p}^{k}-\sum_{k \in K_{1}} \sum_{\left(i, i^{\prime}, t, p\right) \in A^{\prime}} q_{i}^{k} a_{i, i^{\prime}, t, p}^{k} .
$$

Here, $Q$ is the total passenger demand. The second term on the right-hand side of equation (18) is the sum of the loading capacities of the original trains, and the third term on the right-hand side of equation (18) is the sum of the loading capacities of the additional trains.

The two objectives that are specified above may conflict with each other in the scheduling process; that is, more additional trains and stops can decrease the number of unsatisfied passengers but inevitably increase the total travel times. To resolve this potential conflict, we use a linear weighting method to trade off between these two conflicting objectives. Due to the difference in the dimensions of the travel times and passenger demand, we normalize the two objective functions:

$$
\begin{gathered}
\bar{T}_{\text {total }}=\frac{T_{\text {total }}-T_{\text {total }}^{\text {min }}}{T_{\text {total }}^{\max }-T_{\text {total }}^{\min }}, \\
\bar{Q}_{d}=\frac{Q_{d}-Q_{d}^{\min }}{Q_{d}^{\max }-Q_{d}^{\min }},
\end{gathered}
$$

where $\bar{T}_{\text {total }}$ and $\bar{Q}_{d}$ are the normalized values of $T_{\text {total }}$ and $Q_{d}$, respectively; $T_{\text {total }}^{\min }$ and $Q_{d}^{\mathrm{min}}$ are the minimum values of $T_{\text {total }}$ and $Q_{d}$, respectively, under constraints (3)-(16); and $T_{\text {total }}^{\max }$ and $Q_{d}^{\max }$ are the maximum values of $T_{\text {total }}$ and $Q_{d}$, respectively. Based on the discussion above, we model the linear weighted objective function as follows:

$$
F=\theta_{1} \bar{T}_{\text {total }}+\theta_{2} \bar{Q}_{d}
$$

where $\theta_{1}$ and $\theta_{2}$ are the prespecified weights of these two normalized objective functions. According to the practical operation scenario, we empirically determine two suitable parameters to obtain the optimal temporary plan, in which the total travel times and the difference between the total passenger demand and the total loading capacity is minimized. 
3.5. Complexity of the Model. Two types of binary decision variables are proposed in this model: The decision variables of the first type, namely, $x_{i^{\prime}, i+1, t, l}^{k}$ and $x_{i, i^{\prime}, t, p}^{k}$, determine spacetime paths of additional trains in the space-time network. The decision variables of the other type, namely, $y^{k}$, indicate whether each additional train is added or not, which are defined to formulate constraints (3), (4), (15), and (16). In addition, all constraints in the model are linear equalities or inequalities. The objective function of this model is a combination of two normalized linear objective functions with linear weighting. Consequently, our model is a multiobjective 0-1 integer linear programming model.

In the following, the complexity of the model is discussed. The total numbers of decision variables and constraints are listed in Table 2, where the values are the possible maximum values. According to Table 2, the complexity of this model depends on the number of stations on the rail corridor $|S|$, the numbers of time intervals $|T|$, the expected number of additional trains $\left|K_{2}\right|$, the number of types of trains $|L|$, and the number of stopping plans $|P|$.

An example is presented to illustrate the complexity of this model more concretely. Five additional trains on the rail corridor with 20 stations, 2 types of trains, and 2 stopping plans for each train on each segment are considered in these experiments. When a space-time network with a time horizon of 250 timestamps is constructed, there are 47,500 variables with respect to $x_{i^{\prime}, i+1, t, l}^{k}, 50,000$ variables with respect to $x_{i, i^{\prime}, t, p}^{k}$ and 5 variables with respect to $y^{k}$. This results in a large-scale 0-1 integer linear programming model with a total of 97,505 binary decision variables.

\section{Numerical Experiments}

In this section, several numerical experiments are conducted to evaluate the effectiveness and efficiency of our proposed model, and the IBM ILOG CPLEX 12.5 solver is used to solve the 0-1 interprogramming model. All experiments run on a computer with an Intel Core i7 4790K CPU and 8 G RAM.

4.1. Small-Scale Case Study. In this case study, we consider an inbound single-track rail corridor with 9 stations and 8 segments; see Figure 8 . These stations are numbered consecutively from 1 to 9 along the same inbound direction, and two types of high-speed trains that differ in terms of speed, namely, $G$-trains and $D$-trains, are considered in this experiment. The link travel times of the two types of highspeed trains on each segment are presented in Figure 8. For simplicity, the dwelling times of all trains at each station are set to 2 minutes. The minimum departure and arrival headways are both set to 2 minutes to ensure the safe operation of additional trains and to ensure that the operation of the original trains is not disturbed.

In this experiment, there are also 6 original $G$-trains, which are labeled from $G 1$ to $G 6$ according to their departure time and type, in the off-peak period, and the resulting operation plan is presented in Figures 9 and 10. The loading capacity of each train at each station is listed in Table 3. The passenger demand in the peak period and the supply capacity of the original trains in off-peak period at each station are also listed in Table 3. To satisfy as much of the passenger demand in the peak period as possible, in this case, the expected number of additional trains is 5 according to the passenger demand at the origin; see equation (1). In addition, the maximum capacity of each train that is considered in this experiment is assumed to be 400 , and we set the minimal number of $D$-trains to $N_{\min }^{D}$. The value of the attendance rate for each train should fall within the range of $[0.9,1.2]$ to avoid the waste of transport capacity and to improve the service. In this experiment, the time interval $\delta$ in the space-time network is set to 1 minute, and we conduct this experiment with a time horizon of $[0,160]$ minutes. The weight coefficients of the objective function are set to 0.1 and 0.9 , namely, $\theta_{1}=0.1$ and $\theta_{2}=0.9$.

Based on the discussion above, we design codes on the MATLAB platform for obtaining the optimal solution by using the CPLEX solver. According to the resulting optimal solution, the objective value of the total travel time $T_{\text {total }}$ is 476 , and the value of the unsatisfied passenger demand $Q_{d}$ is 245. The optimal timetable and stopping plan for the trains are presented in Figure 9, in which the black lines indicate original trains and the red lines denote additional trains. In Figure 10, the solid dots denote the train stopping at the corresponding station for passenger boarding/alighting, while the hollow dots indicate that the train does not stop at that station. The supply capacity for each station in the peak period that is derived from the resulting optimal solution is listed in Table 3. As a result, as presented in Figure 9, a temporary operation plan is obtained by adding four additional trains in the peak period. In addition, departure and arrival time intervals of these additional trains ensure their safe operation and noninterference with the operation of the original trains.

In this case, according to the passenger demand at the origin, the expected number of additional trains is set to 5 . However, according to the resulting optimal solution, only four additional trains are added into the operation plan, which can balance the total travel times and the passenger demand. These four additional trains lead to an increase in the level of the satisfaction of the passenger demand from $54 \%$ to $95 \%$. However, one more additional train will not only increase the total travel times of trains but also decrease the attendance rates of the trains, thereby resulting in wasting of the transport resources of the rail company. The number of additional trains that is derived from the resulting optimal solution differs from the expected number of additional trains at times because the optimal number of additional trains is influenced by the passenger demand not only at the origin station but also at the intermediate stations. In addition, according to Figure 10, the passenger demand at the intermediate stations influences the stopping plan for the additional trains. All additional trains are scheduled to stop at two stations; namely, station 2 and station 3, to provide sufficient loading capacity because the passenger demands of stations 2 and 3 are much larger than those of the other stations. At the other stations, not all trains are scheduled to stop; for example, only $D 9$ is scheduled to stop at station 5 . 
TABLE 2: Numbers of variables and constraints in model.

\begin{tabular}{lr}
\hline Variable or constraints & Total number at most \\
\hline Binary decision variable $x_{i^{\prime}, i+1, t, l}^{k}$ & $(|S|-1) \cdot|T| \cdot|L| \cdot\left|K_{2}\right|$ \\
Binary decision variable $x_{i, i^{\prime}, t, p}^{k}$ & $|S| \cdot|T| \cdot|P| \cdot\left|K_{2}\right|$ \\
Binary decision variable $y^{k}$ & $\left|K_{2}\right|$ \\
Unique path constraint (3) & $(|S|-1) \cdot\left|K_{2}\right|$ \\
Unique path constraint (4) & $|S| \cdot\left|K_{2}\right|$ \\
Unique path constraints (5) and (6) & $2 \cdot|S| \cdot\left|K_{2}\right| \cdot|T|$ \\
Departure and arrival headway constraints (7) and (8) & $2 \cdot(|S|-1) \cdot|T|$ \\
Tracking headway constraint (9) & $(|S|-1) \cdot|T|$ \\
Train type constraint (10) & $(|S|-2) \cdot|L| \cdot\left|K_{2}\right|$ \\
Train type constraint (11) & $|S|$ \\
Loading capacity constraint (12) & $|S|$ \\
Loading capacity constraints (13) and (14) & $2 \cdot\left|K_{2}\right|$ \\
Loading capacity constraints (15) and (16) & $2 \cdot\left|K_{2}\right|$ \\
\hline
\end{tabular}

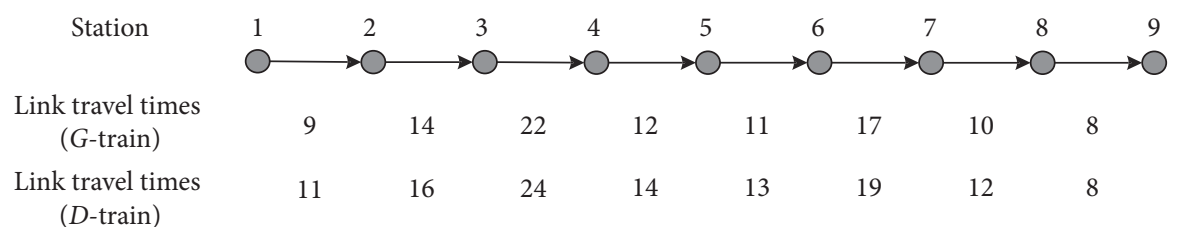

FIGURE 8: A one-way single-track rail corridor.

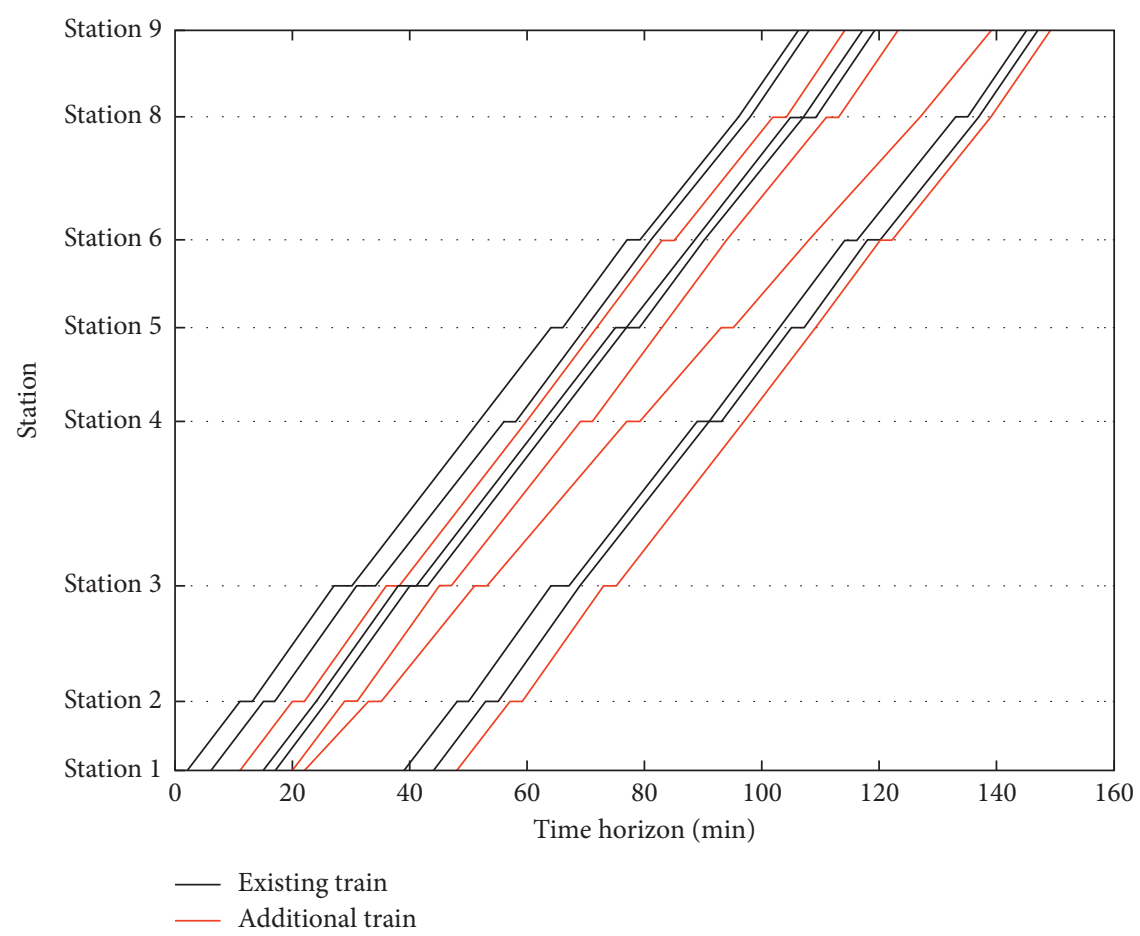

FIGURE 9: Timetable for trains in the small-scale experiment.

4.2. Large-Scale Experiments on the Wuhan-Guangzhou HighSpeed Rail Corridor. In this study, a large-scale experiment on the operational environment of the Wuhan-Guangzhou high-speed railway corridor in China is conducted in which a temporary plan is designed for additional trains in the peak passenger demand period. The Wuhan-Guangzhou highspeed railway corridor is the first high-speed railway corridor in which the trains' maximum velocity is $350 \mathrm{~km} / \mathrm{h}$. The corridor consists of 18 stations and 17 segments, and it is $1069 \mathrm{~km}$ in length, see Figure 11. 


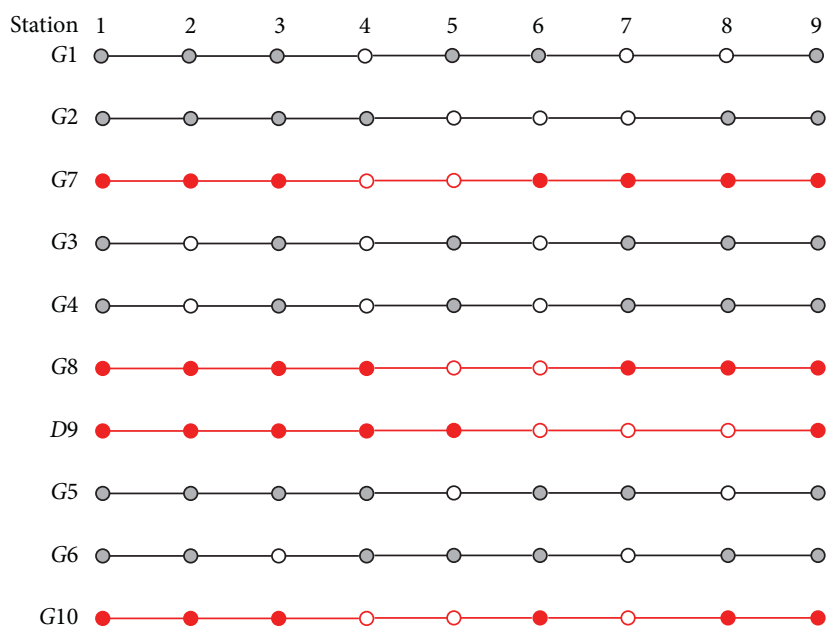

Figure 10: Stop-plans for trains in the small-scale experiment.

TABLE 3: Loading capacity, passenger demand, and supply capacity (unit: person).

\begin{tabular}{lcccccccccc}
\hline Station & 1 & 2 & 3 & 4 & 5 & 6 & 7 & 8 & 9 & Total \\
\hline Loading capacity of each train & 100 & 60 & 95 & 60 & 90 & 50 & 70 & 75 & - & - \\
Passenger demand in peak period & 1100 & 540 & 900 & 300 & 460 & 260 & 370 & 525 & - & 4455 \\
Supply capacity in off-peak period & 600 & 240 & 475 & 180 & 360 & 150 & 210 & 300 & - & 2515 \\
Supply capacity in peak period & 1000 & 480 & 855 & 300 & 450 & 250 & 350 & 525 & - & 4210 \\
\hline
\end{tabular}

4.2.1. Basic Experiment on the Wuhan-Guangzhou HighSpeed Rail Corridor. The experiment is conducted within the time horizon of $[0,600]$ minutes on the WuhanGuangzhou high-speed rail corridor, and two types of trains, namely, $G$-trains and $D$-trains, are considered in these experiments. The length of each segment and the travel times of these two types of trains are listed in Table 4. For simplicity, the dwelling times of the trains are set to 4 minutes at Changsha South station and to 2 minutes at the other stations.

In this study, we discuss passenger demand only from a macroscopic level, and we do not track the number of passengers who get on and off. The daily passenger demand at the station $i$ is derived from historical data on the daily origin-destination (OD) passenger flow [28], which equals the sum of the OD passenger flow from station $i$ to other stations. In addition, since only high-speed trains, namely, $G$-trains and $D$-trains, are scheduled and we set the time horizon to within $[0,600]$ minutes in this experiment, we multiply the daily passenger demand that is derived from the historical data at each station by a coefficient of 0.5 . In addition, we set the passenger demand at Wulongquan East, Lechang East, and Yingde West stations to 0 since the passenger flow is omitted from the historical data [28]. The passenger demand at each station is listed in Table 5.

The rolling stocks on the Wuhan-Guangzhou high-speed rail corridor are mainly CHR380 or CR400, which are typically composed of 16 vehicles or 8 vehicles. To fully utilize the transportation capacity, we assume that all additional trains are composed of 16 vehicles and that each additional train has a maximum loading capacity of 800 people in these experiments. Furthermore, according to the level of the station and the historical data on the passenger demand of each station, the loading capacity of each train at each of these stations is assigned; see Table 5.

We design 20 original trains, namely, 19 G-trains and 1 $D$-train, in the off-peak period from Wuhan to Guangzhou North in the time horizon of $[0,600]$ minutes in this experiment. The supply capacities of these original trains at each station are listed in Table 5. However, these original trains are far from sufficient for providing a capacity that satisfies the passenger demand at each station in the peak period. According to the passenger demands and the loading capacities in Table 5, we expect to add five additional trains to these original trains based on equation (1). To provide passengers with more options, at least one $D$-train must be included in these additional trains, namely, $N_{\min }^{D}=1$. The minimum arrival and departure headways in this experiment are both set to 4 minutes, namely, $h_{\text {amin }}=2 \delta$ and $h_{d \text { min }}=2 \delta$. The weight coefficients of the total travel times and the difference between the total passenger demand and the total loading capacity are set to $\theta_{1}=0.1$ and $\theta_{2}=0.9$, respectively. To fully utilize the trains' capacities, we set the maximum attendance rate and the minimum attendance rate as $\mu_{\max }=1.2$ and $\mu_{\text {min }}=0.9$, respectively.

An experiment is conducted on the MATLAB platform with the CPLEX solver to obtain the optimal temporary operation plan for additional trains in the peak period on the Wuhan-Guangzhou rail corridor. According to the resulting optimal solution, a total of 5 additional trains, with the optimal objective value of 893.1 , should be added into the operation plan. Under the optimal objective value, the total travel time is 1212 minutes, and the difference between the 


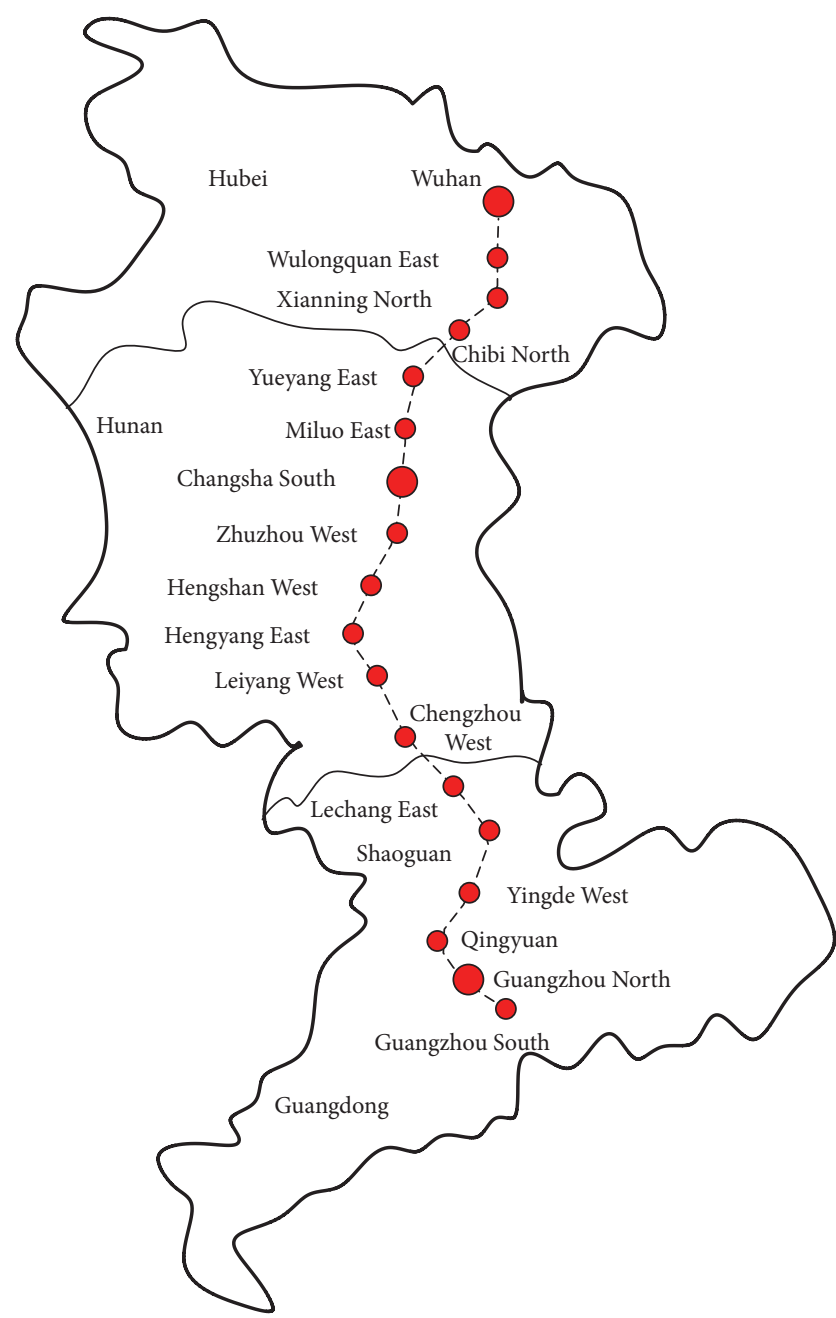

Figure 11: Map of Wuhan-Guangzhou high-speed railway corridor.

TABLE 4: Length and travel times of each segment.

\begin{tabular}{|c|c|c|c|}
\hline \multirow[t]{2}{*}{ Segment } & \multirow{2}{*}{$\begin{array}{l}\text { Length } \\
(\mathrm{km})\end{array}$} & \multicolumn{2}{|c|}{$\begin{array}{c}\text { Travel times } \\
\text { (min) }\end{array}$} \\
\hline & & $G$-train & $D$-train \\
\hline Wuhan-Wulongquan East & 47 & 10 & 12 \\
\hline $\begin{array}{l}\text { Wulongquan East-Xianning } \\
\text { North }\end{array}$ & 38 & 8 & 10 \\
\hline Xianning North-Chibi North & 43 & 8 & 10 \\
\hline Chibi North-Yueyang East & 87 & 18 & 22 \\
\hline Yueyang East-Miluo East & 70 & 14 & 18 \\
\hline Miluo East-Changsha South & 77 & 16 & 20 \\
\hline Changsha South-Zhuzhou West & 52 & 10 & 12 \\
\hline Zhuzhou West-Hengshan West & 84 & 18 & 22 \\
\hline Henshan West-Hengyang East & 41 & 8 & 10 \\
\hline Hengyang East-Leiyang West & 55 & 12 & 14 \\
\hline Leiyang West-Chenzhou West & 98 & 20 & 24 \\
\hline Chenzhou West-Lechang East & 106 & 20 & 26 \\
\hline Lechang East-Shaoguan & 44 & 8 & 12 \\
\hline Shaoguan-Yingde West & 87 & 18 & 22 \\
\hline Yingde West-Qingyuan & 57 & 12 & 14 \\
\hline Qingyuan-Guangzhou North & 36 & 8 & 10 \\
\hline $\begin{array}{l}\text { Guangzhou North-Guangzhou } \\
\text { South }\end{array}$ & 47 & 10 & 12 \\
\hline
\end{tabular}

total passenger demand and the supply capacity is 319 people. The resulting optimal timetable and stopping plan of the original trains and the additional trains are presented in Figures 12 and 13, in which the original trains and the additional trains are numbered according to their departure times from the origin; namely, the original trains from left to right are numbered as $G 1, G 2, \ldots, G 20$, and the additional trains from left to right are labeled as G21, G22, D23, G24, and G25. The supply capacities of the trains that are derived from this operation plan at each station are listed in Table 5.

According to the resulting optimal solution, all additional trains can maintain safety departure and arrival headways at each station of the rail corridor. In addition, they do not disturb the operations of the original trains; see Figures 12 and 13. Since the link travel times of $D$-trains are longer than those of $G$-trains, only one $D$-train is among the additional trains, which facilitates the minimization of the total travel times of all additional trains. In addition, as discussed above, the passenger demands at Wuhongquan East, Lechang East, and Yingde West stations are set to zero in our experiments, and none of the additional trains stop at these three stations, which further decreases the total travel times of the additional trains. According to the results, the stopping plan of these additional trains is mainly influenced by the passenger demand, for example, all five additional trains stop at Changsha station due to its huge passenger demand, while none of the additional trains stop at Hengshan West station.

The total passenger demand of this rail corridor in the peak period is 19065 (see Table 5), and the original trains only offer a capacity of 14676 . The five additional trains add a capacity of 4071 , which satisfies approximately $98.3 \%$ of the passenger demand along this rail corridor. Although the operation plan can satisfy most of the passenger demand, there are two stations, namely, Yueyang East and Chenzhou West, to which sufficient capacity is not provided. As a result, a minority of the passengers, namely, approximately 319 passengers, must travel to their destinations by taking or transferring to short-trip trains.

\subsubsection{Additional Experiments on the Wuhan-Guangzhou} High-Speed Rail Corridor. Several additional experiments are conducted by adopting various parameters on the Wuhan-Guangzhou high-speed rail corridor to further evaluate the performance of our model. Unless stated otherwise, the parameters are the same as for the experimental data in Section 4.2.1.

(1) Additional Experiment with Respect to the Attendance Rate Constraints. In this paper, two objective functions on the travel times and passenger demand are considered, which partially avoids the wasting of the loading capacity of the additional trains. In addition, better control over the attendance rates of trains can provide better service for passengers and improve the efficiency of the utilization of the transportation resource. In this study, to further examine the influences of the attendance rate constraints, an experiment in which the attendance rate is not considered is conducted. 
TABLE 5: Loading capacities, passenger demand, and supply capacity (unit: person).

\begin{tabular}{lcccc}
\hline Station & $\begin{array}{c}\text { Loading capacity } \\
\text { of each train }\end{array}$ & $\begin{array}{c}\text { Passenger demand } \\
\text { in peak period }\end{array}$ & $\begin{array}{c}\text { Supply capacity of } \\
\text { existing trains }\end{array}$ & $\begin{array}{c}\text { Supply capacity of the } \\
\text { new operation plan }\end{array}$ \\
\hline Wuhan & 205 & 4500 & 3600 & 4500 \\
Xianning North & 50 & 175 & 100 & 150 \\
Chibi North & 40 & 160 & 40 & 160 \\
Yueyang East & 187 & 1500 & 1200 & 1440 \\
Miluo East & 100 & 225 & 5200 & 200 \\
Changsha South & 293 & 6500 & 600 & 6500 \\
Zhuzhou West & 125 & 1000 & 400 & 1000 \\
Hengshan West & 100 & 400 & 1000 & 400 \\
Hengyang East & 150 & 1250 & 280 & 340 \\
Leiyang West & 170 & 375 & 1120 & 350 \\
Chenzhou West & 160 & 1000 & 135 & 140 \\
Shaoguan & 200 & 1500 & 210 & 180 \\
Qingyuan & 100 & 200 & 14675 & 280 \\
Guangzhou North & 70 & 280 & 19065 & 18746 \\
Total & - & & & \\
\hline
\end{tabular}

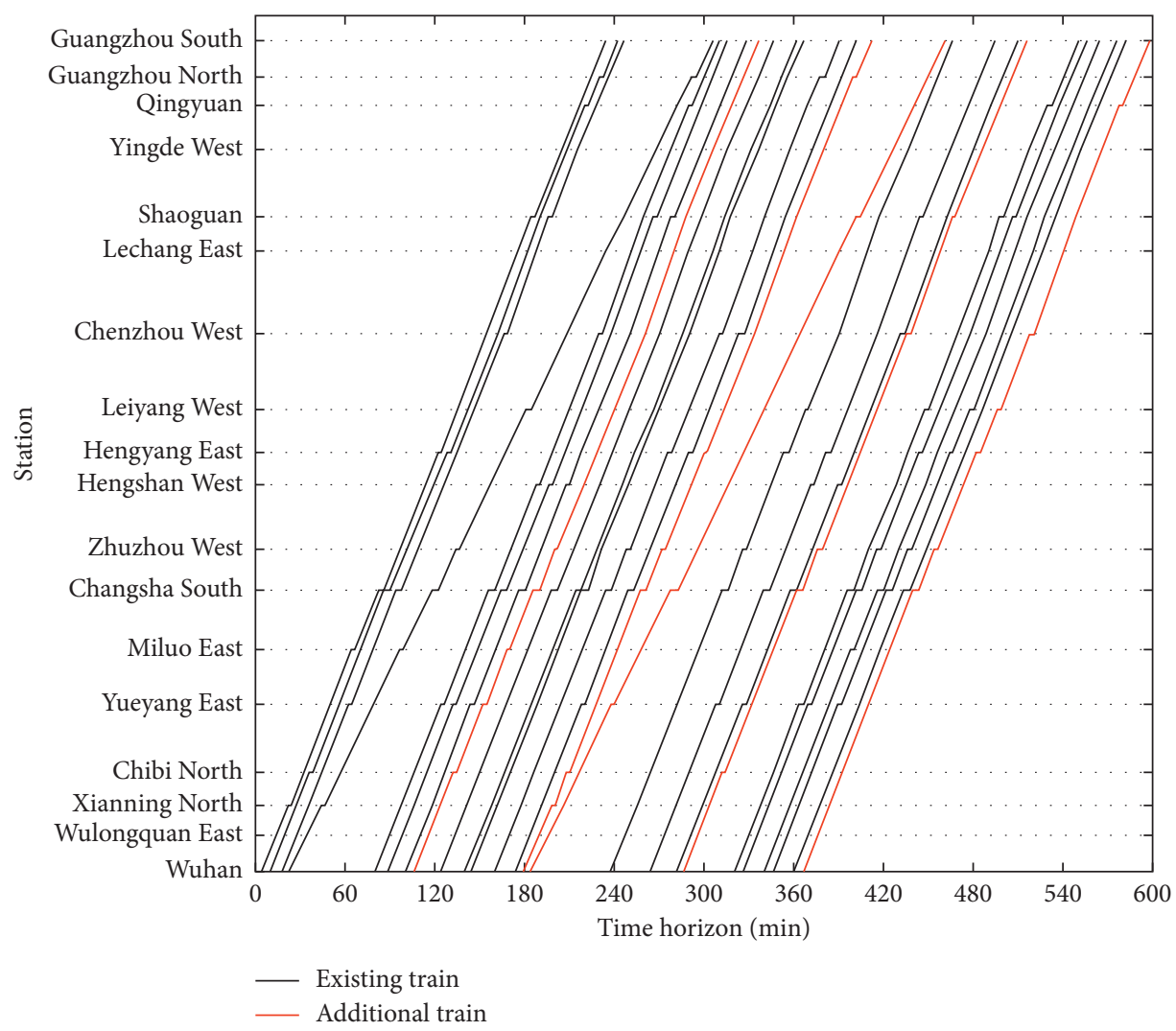

FIGURE 12: The timetable of trains in the basic experiment.

According to the optimal solution of this experiment, five additional trains remain to be added. The total travel time is 1212 minutes, and the difference between the total passenger demand and the supply capacity is 319 people. To demonstrate the difference between this experiment and the previous basic experiment, the timetable and the stopping plan of this experiment are displayed in Figures 14 and 15. Statistical analyses of each additional train that is added in the previous basic experiment and in this experiment are presented in Table 6.
Compared with the previous basic experiment, the number of additional trains and the objective value that is derived from the resulting optimal solution of this experiment do not change. However, differences are identified between these two operation plans, especially in the stopping plans. The loading capacity or attendance rate can be used to measure the efficiency of the stopping plan. Overload leads to poor services, while low-load typically results in a waste of available resources. The results demonstrate that the 


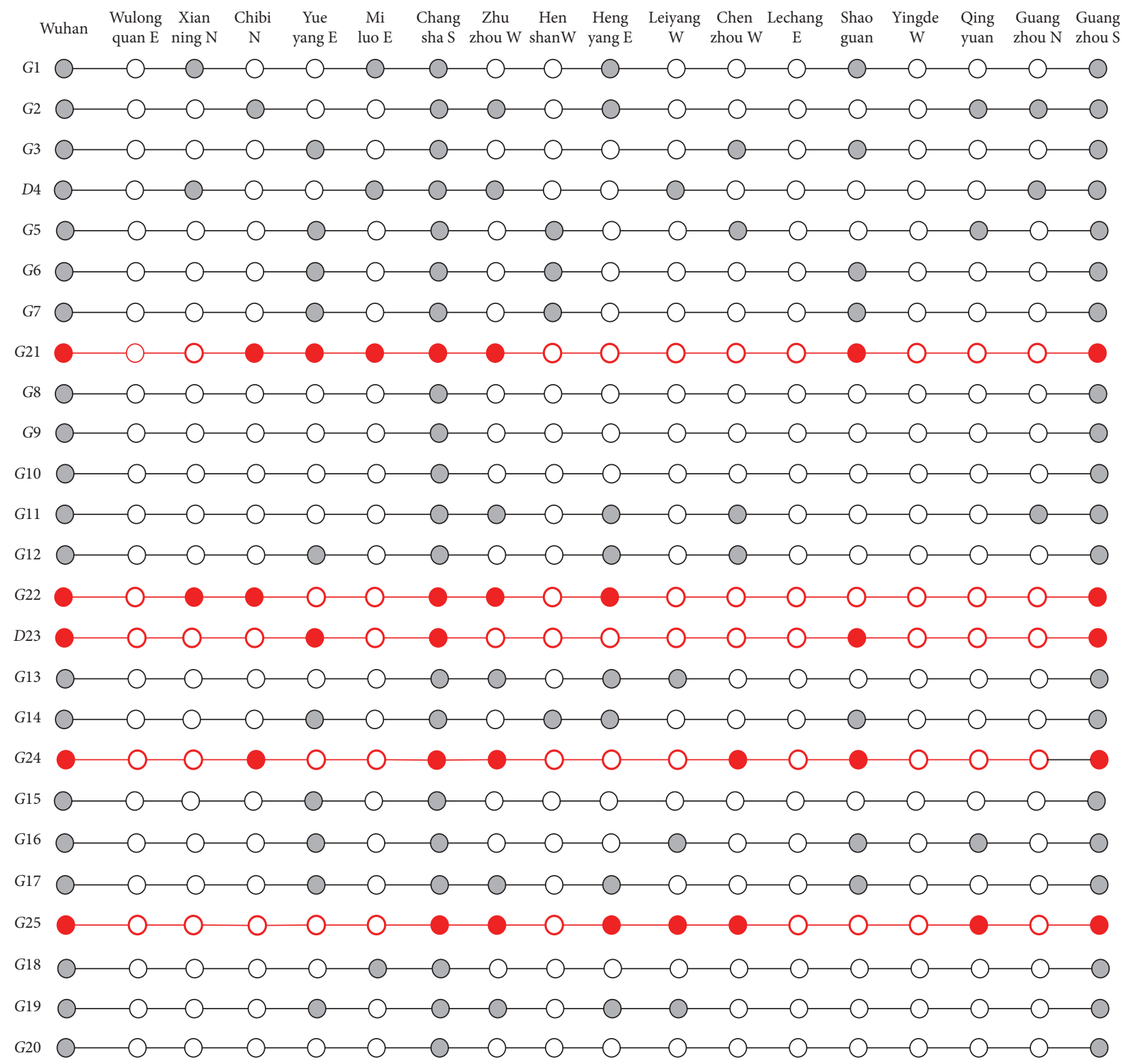

FIgURE 13: The stop-plans of trains in the basic experiment.

stopping plans of the additional trains in this experiment are unreasonable; see Table 6. For example, the loading capacity of G23 is 1108, while the loading capacity of D22 is only approximately 480. In addition, overload enviably leads to longer dwelling times. For example, the dwelling time of G23 is 12 minutes longer than that of D22 in this experiment.

A comparison of the loading capacity and the attendance rate between these two experiments shows the impact of the attendance rate constraint on the stopping plan; see Figure 16. As shown in Figure 16, the stopping plan in the previous basic experiment is more balanced, in which the loading capacities of additional trains are much closer to the maximum capacity of 800 , and the attendance rates are much closer to 1 . The phenomenon supports the efficacy of imposing the attendance rate constraint to increase the efficiency of stopping plan and to generate a more reasonable operation plan that balances the loading capacities of the additional trains.
(2) Additional Experiments with Respect to the Weight Coefficients. $\theta_{1}$ and $\theta_{2}$. In this section, we examine the influences of the weight coefficients $\theta_{1}$ and $\theta_{2}$ in objective function on the performance of the proposed model. We set $\theta_{1}$ and $\theta_{2}$ to $\{0,1.0\},\{0.1,0.9\},\{0.2,0.8\},\{0.3,0.7\},\{0.4,0.6\}$, $\{0.5,0.5\},\{0.6,0.4\},\{0.7,0.3\},\{0.8,0.2\},\{0.9,0.1\}$, and $\{1.0,0\}$ in these experiments. To facilitate the examination of the influence of the weight coefficients, we set the maximum attendance rate to $\mu_{\max }=1.5$ and the minimum attendance rate to $\mu_{\min }=0.7$ in these experiments. The results of these experiments are presented in Table 7.

The first two terms, namely, $T_{\text {total }}$ and $Q_{d}$, which denote the total travel time of the additional trains and the unsatisfied passenger demand, of the objective function are important indicators for evaluating the efficiency of the operation plan. The values of $T_{\text {total }}$ and $Q_{d}$ with the various weight coefficient values are listed in Table 7 , according to 


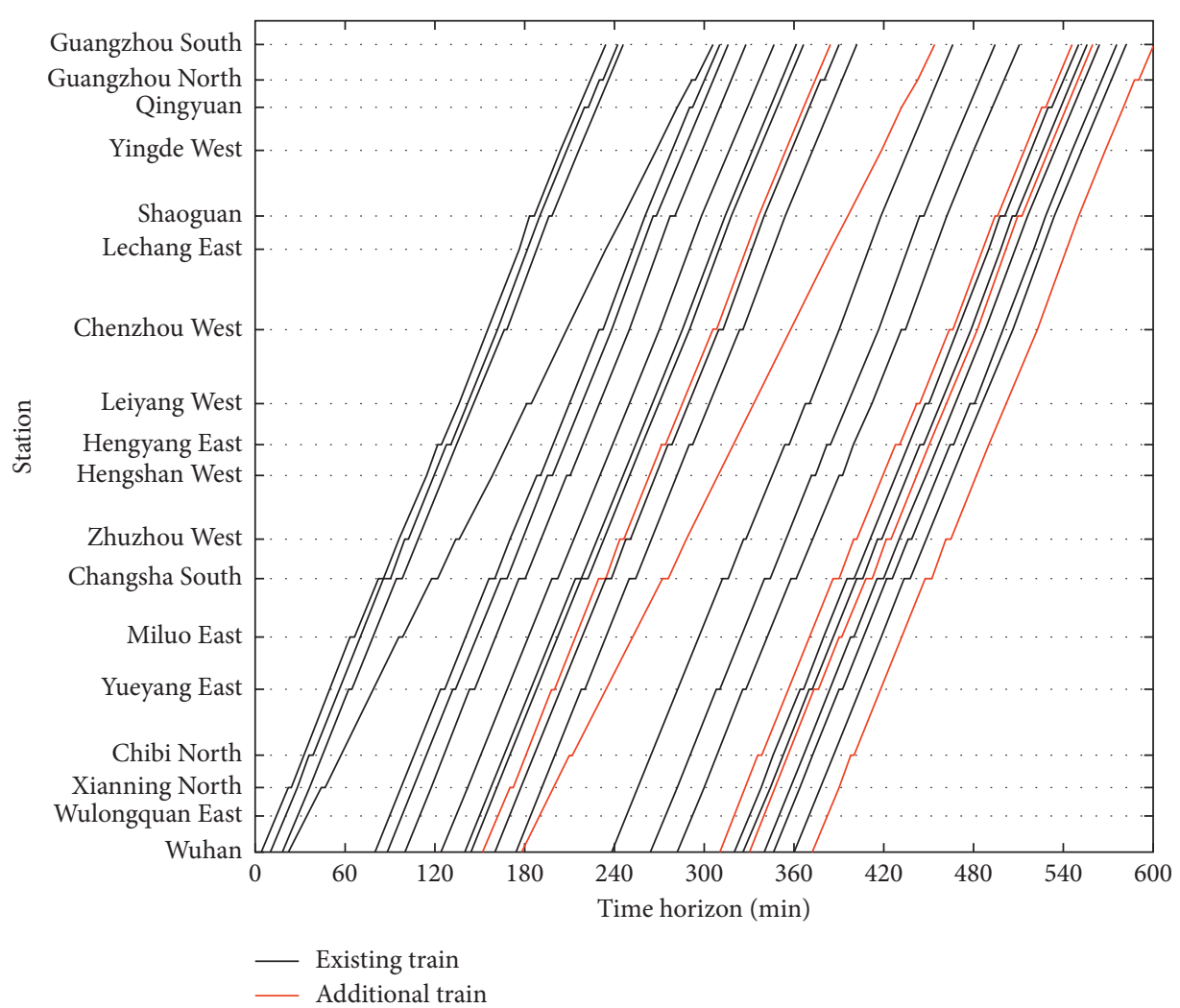

FIgURE 14: The timetable of trains in the experiment without attendance constraints.

which $T_{\text {total }}$ and $Q_{d}$ display various tendencies as functions of $\left\{\theta_{1}, \theta_{2}\right\}$. For instance, when $\left\{\theta_{1}, \theta_{2}\right\}$ varies from $\{0.4,0.6\}$ to $\{0.5,0.5\}, T_{\text {total }}$ decreases from 381 to 262 minutes, while $Q_{d}$ increases from 1299 to 2124 people, respectively.

The weight coefficients determine the strategy that is applied in decision-making. In the experiment, the weight coefficients significantly influence the number of additional trains in the optimal solution.

Since more additional trains can provide a larger capacity to improve the level of satisfaction of the passenger demand while increasing the total travel times, the number of additional trains depends on the parameter pair $\left\{\theta_{1}, \theta_{2}\right\}$. According to Table 7 , when $\theta_{1}$ varies from 0.2 to 0.3 , the number of additional trains decreases from 5 to 4 . Meanwhile, the total travel time decreases from 606 to 494 minutes, and the number of unsatisfied passengers increases from 319 to 759 people. Moreover, when $\theta_{1}$ is set to $0.6,0.7$, $0.8,0.9$, or 1.0 , only one additional train can be added into the operation plan, which satisfies the minimum required number of $D$-trains. When $\left\{\theta_{1}, \theta_{2}\right\}$ is set to $\{0.4,0.6\}$ and $\{0.5,0.5\}$, the unsatisfied passenger demands are 759 and 1299 people, respectively, with 4 additional trains, which demonstrates that the weight coefficients influence not only the number of additional trains but also the stopping plan. Hence, the results of these experiments demonstrate that the weight coefficients significantly affect the operation plan.

In addition, from Table 7, the weight coefficients significantly influence the variation of the computation times. The computation times of the CPLEX solver are approximately $3,000 \mathrm{~s}$ for most cases except for $\left\{\begin{array}{ll}0.2 & 0.8\end{array}\right\}$, for which the computation time is $20,000 \mathrm{~s}$. Thus, a heuristic algorithm with faster arithmetic speed should be designed in our future research.

(3) Additional Experiments with Respect to the Minimum Number of. $D$-Trains $N_{\min }^{D}$. As two types of high-speed trains that differ in terms of velocity, $G$-trains and $D$-trains are considered. It is too necessary to investigate the influences of various values of $N_{\min }^{D}$ on the experimental results. We conduct experiments under the condition that the minimum number of $D$-trains is $1,2,3,4$, or 5 . The attendance rate is required to be within $\left[\begin{array}{ll}0.7 & 1.5\end{array}\right]$ in this set of experiments. Table 8 lists the experimental results for various values of $N_{\min }^{D}$.

Because two types of high-speed trains that differ in terms of velocity, namely, $G$-trains and $D$-trains, are considered in this study, it is necessary to investigate the influences of various values of $N_{\min }^{D}$ on the operation plans. We conduct experiments under the condition that the value of $N_{\min }^{D}$ is set to $1,2,3,4$, or 5 . In addition, the attendance rate is set to within the range of $\left[\begin{array}{ll}0.7 & 1.5\end{array}\right]$ in this set of experiments. The weight coefficients of the total travel times and the passenger demand in these experiments are set to 0.1 and 0.9 , respectively. The experimental results are presented in Table 8.

The results demonstrate that the number of $D$-trains, $N_{D}$, that is derived from the resulting optimal solution is equal to the minimum number of $D$-trains, $N_{\min }^{D}$, that were required in the model. The reason is that the longer link travel times of the $D$-trains would increase the total travel times of additional trains as the number of $D$-trains 


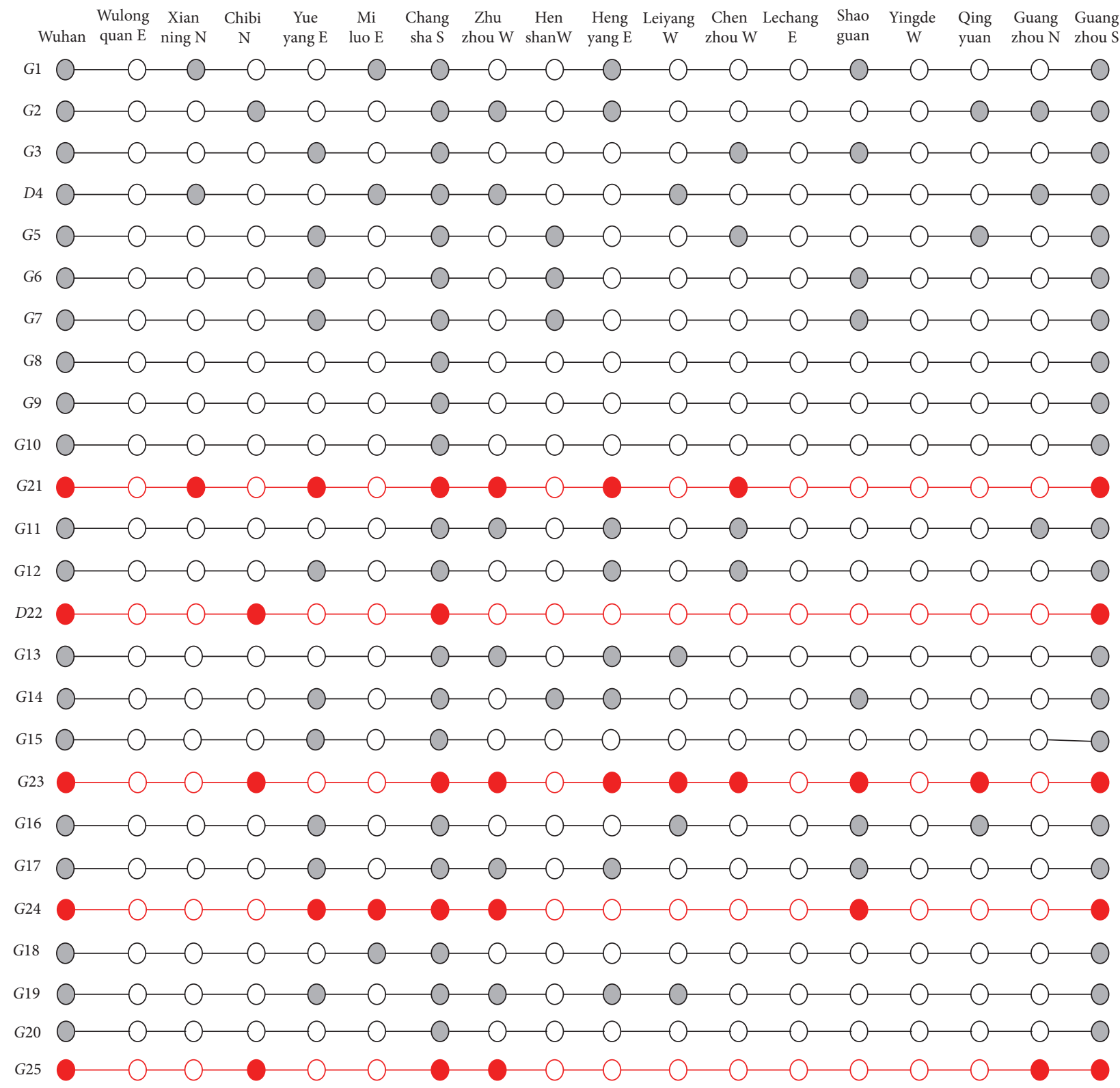

FIGURE 15: The stop plan of trains in the experiment without attendance constraints.

TABLE 6: Some statistical data of each additional train in the results of two experiments.

\begin{tabular}{|c|c|c|c|c|c|}
\hline $\begin{array}{l}\text { Two } \\
\text { experiments }\end{array}$ & $\begin{array}{l}\text { Additional } \\
\text { trains (min) }\end{array}$ & $\begin{array}{l}\text { Link travel } \\
\text { times (min) }\end{array}$ & $\begin{array}{c}\text { Dwelling } \\
\text { times (min) }\end{array}$ & $\begin{array}{l}\text { Attendance } \\
\text { rate }\end{array}$ & $\begin{array}{c}\text { Loading } \\
\text { capacity (person) }\end{array}$ \\
\hline \multirow{5}{*}{$\begin{array}{l}\text { Basic } \\
\text { experiment }\end{array}$} & G21 & 218 & 12 & 0.936 & 750 \\
\hline & G22 & 218 & 14 & 1.031 & 825 \\
\hline & D23 & 270 & 8 & 0.900 & 720 \\
\hline & G24 & 218 & 12 & 1.085 & 868 \\
\hline & G25 & 218 & 14 & 1.135 & 908 \\
\hline \multirow{5}{*}{$\begin{array}{l}\text { The experiment without } \\
\text { attendance rate constraints }\end{array}$} & G21 & 218 & 14 & 1.2 & 963 \\
\hline & G22 & 270 & 6 & 0.6 & 480 \\
\hline & D23 & 218 & 18 & 1.385 & 1108 \\
\hline & G24 & 218 & 12 & 1.088 & 870 \\
\hline & G25 & 218 & 10 & 0.813 & 650 \\
\hline
\end{tabular}




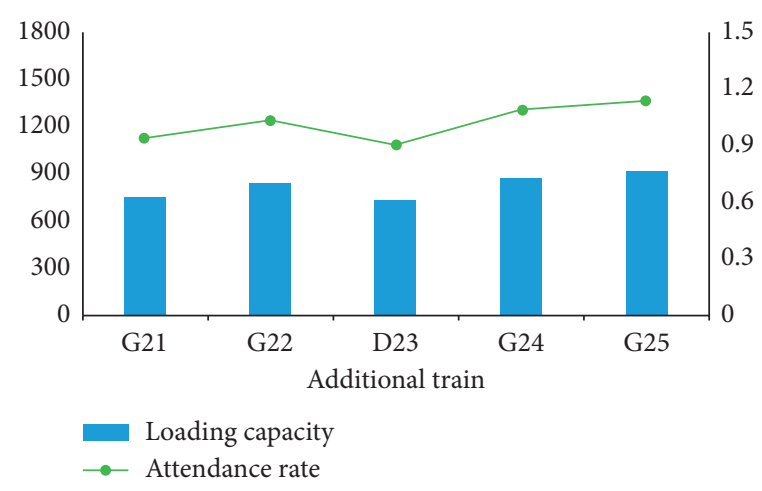

(a)

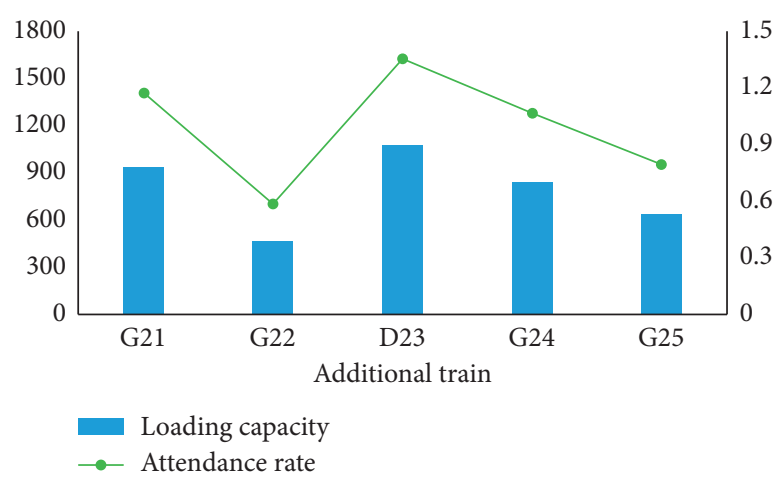

(b)

FIGURE 16: The comparison of loading capacity and the attendance rate of additional trains. (a) Basic Experiment. (b) Experiments without attendance constraints.

TABLE 7: Optimal solutions and computational times with different weight coefficients.

\begin{tabular}{ccccccc}
\hline$\theta_{1}$ & $\theta_{2}$ & $F$ & $N_{A}$ & $T_{\text {total }}$ & $Q_{d}$ & Computational times (s) \\
\hline 0.0 & 1.0 & 319 & 5 & 636 & 319 & 3535.851 \\
0.1 & 0.9 & 893.1 & 5 & 606 & 319 & 3765.045 \\
0.2 & 0.8 & 1467.2 & 5 & 606 & 319 & 18672.136 \\
0.3 & 0.7 & 2013.3 & 4 & 494 & 759 & 3637.107 \\
0.4 & 0.6 & 2303.4 & 4 & 381 & 1299 & 3338.284 \\
0.5 & 0.5 & 2372 & 2 & 262 & 2124 & 3429.896 \\
0.6 & 0.4 & 2184.8 & 1 & 143 & 3317 & 3325.990 \\
0.7 & 0.3 & 1996.1 & 1 & 143 & 3317 & 3462.992 \\
0.8 & 0.2 & 1807.4 & 1 & 143 & 3317 & 3432.277 \\
0.9 & 0.1 & 1618.7 & 1 & 143 & 3317 & 3471.721 \\
1.0 & 0.0 & 1390 & 1 & 139 & 3797 & 3552.153 \\
\hline
\end{tabular}

TABLE 8: Optimal solutions and computational times with value of $N_{\min }^{D}$.

\begin{tabular}{lccccc}
\hline$N_{\min }^{D}$ & $N_{A}$ & $N_{D}$ & $T_{\text {total }}$ & $Q_{d}$ & Computational times (s) \\
\hline 0 & 5 & 0 & 580 & 319 & $3424.896 \mathrm{~s}$ \\
1 & 5 & 1 & 606 & 319 & $3765.045 \mathrm{~s}$ \\
2 & 5 & 2 & 632 & 319 & $3976.685 \mathrm{~s}$ \\
3 & 5 & 3 & 655 & 559 & $5437.791 \mathrm{~s}$ \\
4 & - & - & - & - & - \\
5 & - & - & - & - & - \\
\hline
\end{tabular}

increases. To reduce the total travel times of the additional trains, the optimal number of $D$-trains in these cases should be equal to the minimum number of $D$-trains. In addition, the number of $D$-trains affects the satisfaction of passenger demand. Since $D$-trains have longer link travel times, a satisfactory solution for reducing the total travel times is to reduce the number of stops of the $D$-trains. For instance, the number of stops of two $D$-trains is 8 under the condition of $N_{\text {min }}^{D}=2$, while the number of stops of the three $D$-trains is 6 under the condition of $N_{\min }^{D}=3$. With an increasing number of $D$-trains and a decreasing number of stops of $D$-trains, the number of unsatisfied passengers under the resulting optimal solution also increases. According to $\mathrm{Ta}-$ ble 8 , the number of unsatisfied passengers increases from
319 to 559 as the number of $D$-trains increases from 2 to 3; namely, the number of unsatisfied passengers at Chibi North increases from 0 to 40 and that at Zhuzhou West from 0 to 200.

When the value of $N_{\min }^{D}$ equals 0,1 , and 2, the objective values of the unsatisfied passengers are the same. However, we cannot identify feasible solutions when the value of $N_{\text {min }}^{D}$ is 4 or 5 . This is because compared with the $G$-train travel arc, there would be more travel arcs that are incompatible with the $D$-train travel arc due to its longer travel times. Thus, if the value of $N_{\text {min }}^{D}$ is large, it is difficult to find a feasible path for a $D$-train in the space-time network. Additionally, according to Table 8 , the computation times increase as the value of $N_{\text {min }}^{D}$ increases, which is expected because more $D$-trains would cause more conflicts between $D$-trains and $G$-trains.

\section{Conclusions}

This paper solved the problem of designing an operation plan for additional trains on a high-speed rail corridor. A specialized optimization framework for the design of an operation plan for additional trains is proposed. In the framework, all plans of the number of trains, stop plan, train type, and timetable are jointly optimized. In this paper, we assume that the original trains have priority for rail infrastructures; thus, the operation plan for original trains is fixed, and additional trains cannot disturb the operation of the original trains. To provide sufficient transport capacity, the objective of minimizing the deviation between the passenger demand and the transport capacity was proposed, which was not considered in previous studies on adding trains. Meanwhile, a conflicting objective of minimizing the total travel times of the additional trains to minimize the cost for the railway company was constructed. To obtain the number of additional trains, the train types, the stopping plan, and the timetable for the additional trains simultaneously, several decision variables were introduced, which increase the complexity of the model. By employing a spacetime network diagram and determining the space-time characteristic of the operation plan, we transformed the 
problem of designing the operation plan into a multipletrain path planning problem in a space-time network to increase the efficiency of the modeling. Thus, a biobjective integer linear programming model was constructed. Although we design a primary operation plan, various practical details were considered in this model. For example, headway constraints were imposed to avoid collisions and to avoid disturbing the original trains, and constraints on the attendance rate were imposed to ensure the utilization of the capacity of each train. Two sets of experiments were conducted to evaluate the performance of the method. A small experiment was conducted to evaluate the performance of the proposed approach. In addition, using real data from the Wuhan-Guangzhou rail corridor in China, a set of largescale experiments were conducted to evaluate the applicability of the proposed method. The experimental results demonstrate that the proposed method can be used to obtain a reasonable primary operation plan for additional trains efficiently.

In future research, we will consider this problem on the microlevel. Various operations in stations, for example, train acceleration, deceleration, and overtaking in stations, and the station capacity will be considered. We will also consider the passenger demand on the microlevel and the passenger demand at the origin and destination (OD). In addition, we will extend the model to the high-speed rail network, which will require additional variables and constraints in the model. Thus, the design of an efficient and intelligent algorithm for solving more complicated models is necessary. The efficient design of a more practical and flexible operation plan for additional trains on the rail network will be our research direction.

\section{Data Availability}

Some or all data, models, or code generated or used during the study are available from the corresponding author upon request (list items).

\section{Conflicts of Interest}

The authors declare that there are no conflicts of interest regarding the publication of this paper.

\section{Acknowledgments}

This research was supported by the Fundamental Research Funds for the Central Universities (no. 2018YJS190) and the Research Foundation of State Key Laboratory of Rail Traffic Control and Safety, Beijing Jiaotong University, China (no. RCS2019ZT003).

\section{References}

[1] T. Robenek, Y. Maknoon, S. S. Azadeh, J. Chen, and M. Bierlaire, "Passenger centric train timetabling problem," Transportation Research Part B: Methodological, vol. 89, pp. 107-126, 2016.

[2] A. Caprara, L. G. Kroon, M. Monaci, M. Peeters, and P. Toth, "Passenger railway optimization," in Handbooks in
Operations Research and Management Science, chapter 3, 14, C. Barnhart and G. Laporte, Eds., pp. 129-187, Elsevier, Amsterdam, Netherlands, 2007.

[3] R. M. Lusby, J. Larsen, M. Ehrgott, and D. Ryan, "Railway track allocation: models and methods," OR Spectrum, vol. 33, no. 4, pp. 843-883, 2011.

[4] R. L. Burdett and E. Kozan, "Techniques for inserting additional trains into existing timetables," Transportation Research Part B: Methodological, vol. 43, no. 8-9, pp. 821-836, 2009.

[5] V. Cacchiani, A. Caprara, and P. Toth, "Scheduling extra freight trains on railway networks," Transportation Research Part B: Methodological, vol. 44, no. 2, pp. 215-231, 2010.

[6] Y. Gao, L. Kroon, L. Yang, and Z. Gao, "Three-stage optimization method for the problem of scheduling additional trains on a high-speed rail corridor," Omega, vol. 80, pp. 175-191, 2018.

[7] H. Pouryousef and P. Lautala, "Hybrid simulation approach for improving railway capacity and train schedules," Journal of Rail Transport Planning \& Management, vol. 5, no. 4, pp. 211-224, 2015.

[8] L. Yang, J. Qi, S. Li, and Y. Gao, "Collaborative optimization for train scheduling and train stop planning on high-speed railways," Omega, vol. 64, pp. 57-76, 2015.

[9] M. R. Bussieck, T. Lindner, and M. E. Lübbecke, "A fast algorithm for near cost optimal line plans," Mathematical Methods of Operations Research (ZOR), vol. 59, no. 2, pp. 205-220, 2004.

[10] M. T. Claessens, N. M. van Dijk, and P. J. Zwaneveld, "Cost optimal allocation of rail passenger lines," European Journal of Operational Research, vol. 110, no. 3, pp. 474-489, 1998.

[11] J.-W. Goossens, S. Van Hoesel, and L. Kroon, "A branch-andcut approach for solving railway line-planning problems," Transportation Science, vol. 38, no. 3, pp. 379-393, 2004.

[12] J.-W. Kroon, S. Van Hoesel, and L. Kroon, "On solving multitype railway line planning problems," European Journal of Operational Research, vol. 168, no. 2, pp. 403-424, 2006.

[13] M. R. Bussieck, P. Kreuzer, and U. T. Zimmermann, "Optimal lines for railway systems," European Journal of Operational Research, vol. 96, no. 1, pp. 54-63, 1997.

[14] A. Schöbel and S. Scholl, Line Planning with Minimal Traveling Time, Universität Göttingen, Göttingen, Germany, 2006.

[15] X. Zhou and M. Zhong, "Bicriteria train scheduling for high-speed passenger railroad planning applications," European Journal of Operational Research, vol. 167, no. 3, pp. 752-771, 2005.

[16] Y.-H. Chang, C.-H. Yeh, and C.-C. Shen, "A multiobjective model for passenger train services planning: application to Taiwan's high-speed rail line," Transportation Research Part B: Methodological, vol. 34, no. 2, pp. 91-106, 2000.

[17] D. Chen, S. Ni, C. Xu, H. Lv, and S. Wang, "A soft rough-fuzzy preference set-based evaluation method for high-speed train operation diagrams," Mathematical Problems in Engineering, vol. 2016, Article ID 8763589, 10 pages, 2016.

[18] K. Ghoseiri, F. Szidarovszky, and M. J. Asgharpour, "A multiobjective train scheduling model and solution," Transportation Research Part B: Methodological, vol. 38, no. 10, pp. 927-952, 2004.

[19] Y. J. Lee, "Mathematical modeling for optimizing skip-stop rail transit operation strategy using genetic algorithm," Research Report, Department of Transportation and Urban Infrastructure Studies Morgan State University, Baltimore, MD, USA, 2012.

[20] Y.-J. Lee, S. Shariat, and K. Choi, "Optimizing skip-stop rail transit stopping strategy using a genetic algorithm," Journal of Public Transportation, vol. 17, no. 2, pp. 135-164, 2014. 
[21] H. Niu, X. Zhou, and R. Gao, "Train scheduling for minimizing passenger waiting time with time-dependent demand and skip-stop patterns: nonlinear integer programming models with linear constraints," Transportation Research Part B: Methodological, vol. 76, pp. 117-135, 2015.

[22] E. Hassannayebi, S. H. Zegordi, M. R. Amin-Naseri, and M. Yaghini, "Demand-oriented timetable design for urban rail transit under stochastic demand," Journal of Industrial and Systems Engineering, vol. 9, no. 3, 2016.

[23] S. Li, R. Xu, and K. Han, "Demand-oriented train services optimization for a congested urban rail line: integrating short turning and heterogeneous headways," Transportmetrica A: Transport Science, vol. 15, no. 2, pp. 1459-1486, 2019.

[24] D. Li, T. Zhang, X. Dong, Y. Yin, and J. Cao, "Trade-off between efficiency and fairness in timetabling on a single urban rail transit line under time-dependent demand condition," Transportmetrica B: Transport Dynamics, vol. 7, no. 1, pp. 1203-1231, 2019.

[25] W. Zhou, W. Fan, X. You, and L. Deng, "Demand-oriented train timetabling integrated with passenger train-booking decisions," Sustainability, vol. 11, no. 18, p. 4932, 2019.

[26] U. Brännlund, P. O. Lindberg, A. Nõu, and J.-E. Nilsson, "Railway timetabling using Lagrangian relaxation," Transportation Science, vol. 32, no. 4, pp. 358-369, 1998.

[27] J. Yin, L. Yang, T. Tang, Z. Gao, and B. Ran, "Dynamic passenger demand oriented metro train scheduling with energy-efficiency and waiting time minimization: mixed-integer linear programming approaches," Transportation Research Part B: Methodological, vol. 97, pp. 182-213, 2017.

[28] J. Qi, S. Li, Y. Gao, K. Yang, and P. Liu, "Joint optimization model for train scheduling and train stop planning with passengers distribution on railway corridors," Journal of the Operational Research Society, vol. 69, no. 4, pp. 556-570, 2018. 